\title{
Pacific
}

Journal of

Mathematics

\section{STRONG KÄHLER WITH TORSION STRUCTURES \\ FROM ALMOST CONTACT MANIFOLDS}

MARISA FernándeZ, ANNA Fino,

Luis Ugarte and RaQuel Villacampa 


\title{
STRONG KÄHLER WITH TORSION STRUCTURES FROM ALMOST CONTACT MANIFOLDS
}

\author{
MARISA FERnÁndEZ, ANNA Fino, \\ LUIS UGARTE AND RAQUEL VILLACAMPA
}

\begin{abstract}
For an almost contact metric manifold $N$, we find conditions under which either the total space of an $S^{1}$-bundle over $N$ or the Riemannian cone over $N$ admit a strong Kähler with torsion (SKT) structure. In so doing, we construct new 6-dimensional SKT manifolds. Moreover, we study the geometric structure induced on a hypersurface of an SKT manifold and use it to construct new SKT manifolds via appropriate evolution equations. We also study hyper-Kähler with torsion (HKT) structures on the total space of an $S^{1}$-bundle over manifolds with three almost contact structures.
\end{abstract}

\section{Introduction}

On any Hermitian manifold $\left(M^{2 n}, J, h\right)$ there exists a unique Hermitian connection $\nabla^{B}$ with totally skew-symmetric torsion, which is called the Bismut connection after [Bismut 1989]. The torsion 3-form $h\left(X, T^{B}(Y, Z)\right)$ of $\nabla^{B}$ can be identified with the 3-form

$$
-J d F(\cdot, \cdot, \cdot)=-d F(J \cdot, J \cdot, J \cdot),
$$

where $F(\cdot, \cdot)=h(\cdot, J \cdot)$ is the fundamental 2-form associated to the Hermitian structure $(J, h)$.

Hermitian structures with closed $J d F$ are called strong Kähler with torsion (in short, SKT) or pluriclosed [Egidi 2001]. Since $\partial \bar{\partial}$ acts as $\frac{1}{2} d J d$ on forms of bidegree $(1,1)$, the latter condition is equivalent to $\partial \bar{\partial} F=0$. SKT structures have been recently studied by many authors, and they also have applications in type II string theory and in 2-dimensional supersymmetric $\sigma$-models [Gates et al. 1984; Strominger 1986; Ivanov and Papadopoulos 2001].

The class of SKT metrics includes of course the Kähler metrics, but as in [Fino et al. 2004], we are interested on non-Kähler geometry, so by an SKT metric we

MSC2000: primary 53C55; secondary 53C26, 22E25, 53C15.

Keywords: Hermitian metric, hypercomplex structure, torsion, almost contact structure,

Riemannian cone.

Partially supported through Project MICINN (Spain) MTM2008-06540-C02-01/02, Project MIUR "Riemannian Metrics and Differentiable Manifolds" and by GNSAGA of INdAM. 
will mean a Hermitian metric $h$ whose fundamental 2-form $F$ is $\partial \bar{\partial}$-closed but not $d$-closed.

Gauduchon [1984] showed that on a compact complex surface, an SKT metric can be found in the conformal class of any given Hermitian metric, but in higher dimensions the situation is more complicated.

SKT structures on 6-dimensional nilmanifolds, that is, on compact quotients of nilpotent Lie groups by discrete subgroups, were classified in [Fino et al. 2004; Ugarte 2007]. Simply connected examples of 6-dimensional SKT manifolds have been found in [Grantcharov et al. 2008] by using torus bundles, and recently Swann [2010] has reproduced them via the twist construction, by extending them to higher dimensions and finding new compact simply connected SKT manifolds. Moreover, Fino and Tomassini [2009] showed that the SKT condition is preserved by the blow-up construction.

The odd-dimensional analogue of a Hermitian structure is given by a normal almost contact metric structure. Indeed, on the product $N^{2 n+1} \times \mathbb{R}$ of a $(2 n+1)$ dimensional almost contact metric manifold $N^{2 n+1}$ by the real line $\mathbb{R}$, it is possible to define a natural almost complex structure, which is integrable if and only if the almost contact metric structure on $N^{2 n+1}$ is normal [Sasaki and Hatakeyama 1961]. More generally, it is possible to construct Hermitian manifolds starting from an almost contact metric manifold $N^{2 n+1}$ by considering a principal fiber bundle $P$ with base space $N^{2 n+1}$ and structural group $S^{1}$, that is, an $S^{1}$-bundle over $N^{2 n+1}$; see [Ogawa 1963]. Indeed, by using the almost contact metric structure on $N^{2 n+1}$ and the connection 1-form $\theta$, Ogawa constructed an almost Hermitian structure $(J, h)$ on $P$ and found conditions under which $J$ is integrable and $(J, h)$ is Kähler.

In Section 2, we determine in Theorem 2.3 general conditions under which an $S^{1}$-bundle over an almost contact metric $(2 n+1)$-dimensional manifold $N^{2 n+1}$ is SKT. We study the particular case when $N^{2 n+1}$ is quasi-Sasakian, that is, when it has an almost contact metric structure for which the fundamental form is closed (Corollary 2.4). In this way, we are able to construct some new 6-dimensional SKT examples, starting from 5-dimensional quasi-Sasakian Lie algebras, and also from Sasakian ones.

A Sasakian structure can be also seen as the analogue, in odd dimensions, of a Kähler structure. Indeed, by [Boyer and Galicki 1999], a Riemannian manifold $\left(N^{2 n+1}, g\right)$ of odd dimension $2 n+1$ admits a compatible Sasakian structure if and only if the Riemannian cone $N^{2 n+1} \times \mathbb{R}^{+}$is Kähler. In Section 3, Theorem 3.1 gives the conditions that must be satisfied by the compatible almost contact metric structure on a Riemannian manifold $\left(N^{2 n+1}, g\right)$ in order that the Riemannian cone $N^{2 n+1} \times \mathbb{R}^{+}$be SKT. We provide an example of an SKT manifold constructed as a Riemannian cone, and in Section 4 we consider the case when the Riemannian cone is 6-dimensional. This case is interesting since one can impose that the SKT 
structure is in addition an SKT SU(3)-structure, and one can find relations with the SU(2)-structures studied by Conti and Salamon [2007].

In Section 5, we study the geometric structure induced naturally on any oriented hypersurface $N^{2 n+1}$ of a $(2 n+2)$-dimensional manifold $M^{2 n+2}$ carrying an SKT structure, and in Section 6, we use such structures in Theorem 6.4 to construct new SKT manifolds via appropriate evolution equations [Hitchin 2001; Conti and Salamon 2007] starting from a 5-dimensional manifold endowed with an SU(2)structure.

A good quaternionic analogue of Kähler geometry is given by hyper-Kähler with torsion (in short, HKT) geometry. An HKT manifold is a hyper-Hermitian manifold $\left(M^{4 n}, J_{1}, J_{2}, J_{3}, h\right)$ admitting a hyper-Hermitian connection with totally skew-symmetric torsion, that is, one in which the three Bismut connections associated with the three Hermitian structures $\left(J_{r}, h\right)$ coincide for $r=1,2,3$. This geometry was introduced by Howe and Papadopoulos [1996] and later studied in [Grantcharov and Poon 2000; Fino and Grantcharov 2004; Barberis et al. 2009; Barberis and Fino 2008; Swann 2010].

In the interesting special case in which the torsion 3-form of such a hyperHermitian connection is closed, the HKT manifold is called strong.

In Section 7, Theorem 7.1 gives conditions under which an $S^{1}$-bundle over a $(4 n+3)$-dimensional manifold endowed with three almost contact metric structures is HKT and in particular when it is strong HKT.

\section{SKT structures arising from $S^{1}$-bundles}

Consider a $(2 n+1)$-manifold $N^{2 n+1}$ endowed with an almost contact metric structure $(I, \xi, \eta, g)$; that is, $I$ is a tensor field of type $(1,1), \xi$ is a vector field, $\eta$ is a 1 -form, and $g$ is a Riemannian metric on $N^{2 n+1}$, satisfying together the conditions

$$
I^{2}=-\mathrm{Id}+\eta \otimes \xi, \quad \eta(\xi)=1, \quad g(I U, I V)=g(U, V)-\eta(U) \eta(V)
$$

for any vector fields $U$ and $V$ on $N^{2 n+1}$. Denote by $\omega$ the fundamental 2-form of $(I, \xi, \eta, g)$; that is, $\omega$ is the 2 -form on $N^{2 n+1}$ given by

$$
\omega(\cdot, \cdot)=g(\cdot, I \cdot) \text {. }
$$

Given the tensor field $I$, consider its Nijenhuis torsion $[I, I]$, defined by

$$
[I, I](X, Y)=I^{2}[X, Y]+[I X, I Y]-I[I X, Y]-I[X, I Y] .
$$

On the product $N^{2 n+1} \times \mathbb{R}$, one can define a natural almost complex structure

$$
J\left(X, f \frac{d}{d t}\right)=\left(I X+f \xi,-\eta(X) \frac{d}{d t}\right),
$$

where $f$ is a $\mathscr{C}^{\infty}$-function on $N^{2 n+1} \times \mathbb{R}$ and $t$ is the coordinate on $\mathbb{R}$. 
Definition 2.1 [Sasaki and Hatakeyama 1961]. We call an almost contact metric structure $(I, \xi, \eta, g)$ on $N^{2 n+1}$ normal if the almost complex structure $J$ on $N^{2 n+1} \times \mathbb{R}$ is integrable, or equivalently if

$$
[I, I](X, Y)+2 d \eta(X, Y) \xi=0
$$

for any vector fields $X, Y$ on $N^{2 n+1}$.

By [Blair 1967, Lemma 2.1], one has $i_{\xi} d \eta=0$ for a normal almost contact metric structure $(I, \xi, \eta, g)$.

Remark 2.2. The normality of the almost contact structure implies as well that $I d \eta=d \eta$. Indeed, $d(\eta-i d t)=d \eta$ has no $(0,2)$-part and therefore has no $(2,0)$ part since $d \eta$ is real. Thus, $J d \eta=d \eta$, but $J d \eta=I d \eta$ as well since $i_{\xi} d \eta=0$.

We recall that a Hermitian manifold $(M, J, h)$ is SKT if and only if the 3-form $J d F$ is closed, where $F$ is the fundamental 2-form of $(J, h)$. We will use the convention that $J$ acts on $r$-forms $\beta$ by

$$
(J \beta)\left(X_{1}, \ldots, X_{r}\right)=\beta\left(J X_{1}, \ldots, J X_{r}\right) \text { for any vector fields } X_{1}, \ldots, X_{r} .
$$

We now show general conditions under which an $S^{1}$-bundle over an almost contact metric $(2 n+1)$-dimensional manifold is SKT.

Let $\left(N^{2 n+1}, I, \xi, \eta\right)$ be a $(2 n+1)$-dimensional almost contact manifold, and let $\Omega$ be a closed 2-form on $N^{2 n+1}$ that represents an integral cohomology class on $N^{2 n+1}$. From the well-known result of Kobayashi [1956], we can consider the circle bundle $S^{1} \hookrightarrow P \rightarrow N^{2 n+1}$ and the connection 1-form $\theta$ on $P$ whose curvature form is $d \theta=\pi^{*}(\Omega)$, where $\pi: P \rightarrow N^{2 n+1}$ is the projection.

By using the almost contact structure $(I, \xi, \eta)$ and the connection 1-form $\theta$, one can define an almost complex structure $J$ on $P$ as follows [Ogawa 1963]. For any right-invariant vector field $X$ on $P$, the vector field $J X$ is given by

$$
\theta(J X)=-\pi^{*}\left(\eta\left(\pi_{*} X\right)\right) \text { and } \pi_{*}(J X)=I\left(\pi_{*} X\right)+\tilde{\theta}(X) \xi,
$$

where $\tilde{\theta}(X)$ is the unique function on $N^{2 n+1}$ such that

$$
\pi^{*} \tilde{\theta}(X)=\theta(X) .
$$

This definition can be extended to an arbitrary vector field $X$ on $P$ since $X$ can be written in the form $X=\sum_{j} f_{j} X_{j}$, with $f_{j}$ smooth functions on $P$, and $X_{j}$ right-invariant vector fields. Then $J X=\sum_{j} f_{j} J X_{j}$.

Ogawa [1963] showed that when $\left(N^{2 n+1}, I, \xi, \eta\right)$ is normal, the almost complex structure $J$ on $P$ defined by (2) is integrable if and only if $d \theta$ is $J$-invariant, that is, if $J(d \theta)=d \theta$ or equivalently $d \theta(J X, Y)+d \theta(X, J Y)=0$ for any vector fields $X$ and $Y$ on $P$. That is, $d \theta$ is a complex 2-form on $P$ having bidegree $(1,1)$ with respect to $J$. 
In terms of the 2 -form $\Omega$, whose lift to $P$ is the curvature of the circle bundle $S^{1} \hookrightarrow P \rightarrow N^{2 n+1}$, the previous condition means that $\Omega$ is $I$-invariant, that is, $I(\Omega)=\Omega$. Therefore $i_{\xi} \Omega=0$.

If $\left\{e^{1}, \ldots, e^{2 n}, \eta\right\}$ is an adapted coframe on a neighborhood $U$ on $N^{2 n+1}$, that is,

$$
I e^{2 j-1}=-e^{2 j} \quad \text { and } \quad I e^{2 j}=e^{2 j-1} \quad \text { for } 1 \leq j \leq n,
$$

then we can take $\left\{\pi^{*} e^{1}, \ldots, \pi^{*} e^{2 n}, \pi^{*} \eta, \theta\right\}$ as a coframe in $\pi^{-1}(U)$. By using the coframe $\left\{\pi^{*} e^{1}, \ldots, \pi^{*} e^{2 n}\right\}$, we may write

$$
d \theta=\pi^{*} \alpha+\pi^{*} \beta \wedge \pi^{*} \eta,
$$

where $\alpha$ is a 2 -form in $\bigwedge^{2}\left\langle e^{1}, \ldots, e^{2 n}\right\rangle$, and $\beta \in \bigwedge^{1}\left\langle e^{1}, \ldots, e^{2 n}\right\rangle$.

Suppose that $N^{2 n+1}$ has a normal almost contact metric structure $(I, \xi, \eta, g)$. We consider a principal $S^{1}$-bundle $P$ with base space $N^{2 n+1}$ and connection 1-form $\theta$, and endow $P$ with the almost complex structure $J$ (associated to $\theta$ ) defined by (2). Since $N^{2 n+1}$ has a Riemannian metric $g$, a Riemannian metric $h$ on $P$ compatible with $J$ (see [Ogawa 1963]) is given by

$$
h(X, Y)=\pi^{*} g\left(\pi_{*} X, \pi_{*} Y\right)+\theta(X) \theta(Y)
$$

for any right-invariant vector fields $X$ and $Y$. This definition can be extended to any vector field on $P$.

Theorem 2.3. Consider a $(2 n+1)$-dimensional almost contact metric manifold $\left(N^{2 n+1}, I, \xi, \eta, g\right)$, and let $\Omega$ be a closed 2-form on $N^{2 n+1}$ that represents an integral cohomology class. Consider the circle bundle $S^{1} \hookrightarrow P \rightarrow N^{2 n+1}$ with connection 1-form $\theta$, whose curvature form is $d \theta=\pi^{*}(\Omega)$ for the projection $\pi: P \rightarrow N^{2 n+1}$.

The almost Hermitian structure $(J, h)$ on $P$ defined by (2) and (4) is SKT if and only if $(I, \xi, \eta, g)$ is normal, $d \theta$ is $J$-invariant, and

$$
\begin{aligned}
d\left(\pi^{*}\left(I\left(i_{\xi} d \omega\right)\right)\right) & =0, \\
d\left(\pi^{*}(I(d \omega)-d \eta \wedge \eta)\right) & =\left(-\pi^{*}\left(I\left(i_{\xi} d \omega\right)\right)+\pi^{*} \Omega\right) \wedge \pi^{*} \Omega,
\end{aligned}
$$

where $\omega$ is the fundamental form of the almost contact metric structure $(I, \xi, \eta, g)$. Proof. As we mentioned, a result of Ogawa [1963] asserts that the almost complex structure $J$ is integrable if and only if $(I, \xi, \eta, g)$ is normal and $J(d \theta)=d \theta$. Thus, $(J, h)$ is SKT if and only if the 3-form $J d F$ is closed. Using the first equality in (2), we find that the fundamental 2-form $F$ on $P$ is

$$
\begin{aligned}
F(X, Y) & =h(X, J Y) \\
& =\pi^{*} g\left(\pi_{*} X, \pi_{*} J Y\right)+\theta(X) \theta(J Y) \\
& =\pi^{*} g\left(\pi_{*} X, \pi_{*} J Y\right)-\theta(X) \pi^{*} \eta\left(\pi_{*} Y\right) .
\end{aligned}
$$


Therefore, taking into account that we are working with a circle bundle (whose fiber is thus 1-dimensional), we have

$$
\begin{aligned}
F & =\pi^{*} \omega+\pi^{*} \eta \wedge \theta, \\
d F & =\pi^{*}(d \omega)+\pi^{*}(d \eta) \wedge \theta-\pi^{*} \eta \wedge d \theta, \\
J d F & =J\left(\pi^{*}(d \omega)\right)-J\left(\pi^{*}(d \eta)\right) \wedge \pi^{*} \eta-\theta \wedge d \theta
\end{aligned}
$$

since $J\left(\pi^{*} \eta\right)=\theta$ and $J$ is integrable, and so $J(d \theta)=d \theta$. Moreover,

$$
J\left(\pi^{*}(d \omega)\right)=\pi^{*}(I(d \omega))+\pi^{*}\left(I\left(i_{\xi} d \omega\right)\right) \wedge \theta .
$$

Indeed, locally and in terms of the adapted basis $\left\{e^{1}, \ldots, e^{2 n+1}\right\}$ with

$$
I e^{2 j-1}=-e^{2 j} \quad \text { for } 1 \leq j \leq n, \quad I e^{2 n+1}=0, \quad \text { and } \quad \eta=e^{2 n+1},
$$

we can write $d \omega=\alpha+\beta \wedge \eta$, where the local forms $\alpha \in \bigwedge^{3}\left\langle e^{1}, \ldots, e^{2 n}\right\rangle$ and $\beta \in \bigwedge^{2}\left\langle e^{1}, \ldots, e^{2 n}\right\rangle$ are generated only by $e^{1}, \ldots, e^{2 n}$. Furthermore, we have $I \alpha=I(d \omega)$ and $\beta=i_{\xi} d \omega$. Thus

$$
J\left(\pi^{*}(d \omega)\right)=J\left(\pi^{*}(\alpha)\right)+J\left(\pi^{*}\left(i_{\xi} d \omega\right)\right) \wedge \theta .
$$

Now, by using (2) and (3), we see that $J\left(\pi^{*}(\alpha)\right)=\pi^{*}(I \alpha)$ and $J\left(\pi^{*}\left(i_{\xi} d \omega\right)\right)=$ $\pi^{*}\left(I\left(i_{\xi} d \omega\right)\right)$, which proves (7). As a consequence of Remark 2.2,

$$
J\left(\pi^{*}(d \eta)\right)=\pi^{*}(I(d \eta))-\pi^{*}\left(I\left(i_{\xi} d \eta\right)\right) \wedge \theta=\pi^{*}(d \eta)
$$

since $i_{\xi} d \eta=0$ and $I d \eta=d \eta$.

Using (7) and (8), we get

$$
J d F=\pi^{*}(I(d \omega))+\pi^{*}\left(I\left(i_{\xi} d \omega\right)\right) \wedge \theta-\pi^{*}(d \eta) \wedge \pi^{*} \eta-\theta \wedge d \theta .
$$

Therefore,

$$
\begin{aligned}
d(J d F)=d\left(\pi^{*}(I(d \omega))\right)+d\left(\pi^{*}\left(I\left(i_{\xi} d \omega\right)\right)\right) & \wedge \theta+\pi^{*}\left(I\left(i_{\xi} d \omega\right)\right) \wedge d \theta \\
& -d\left(\pi^{*}(d \eta)\right) \wedge \pi^{*} \eta-\pi^{*}(d \eta) \wedge d \pi^{*} \eta-d \theta \wedge d \theta .
\end{aligned}
$$

Consequently, $d(J d F)=0$ if and only if

$$
\begin{aligned}
d\left(\pi^{*}\left(I\left(i_{\xi} d \omega\right)\right)\right) & =0, \\
d\left(\pi^{*}(I(d \omega)-d \eta \wedge \eta)\right) & =\left(\pi^{*}\left(-I\left(i_{\xi} d \omega\right)\right)+d \theta\right) \wedge d \theta .
\end{aligned}
$$

An almost contact metric manifold $\left(N^{2 n+1}, I, \xi, \eta, g\right)$ is quasi-Sasakian if it is normal and its fundamental form $\omega$ is closed. In particular, if $d \eta=\alpha \omega$, then the almost contact metric structure is called $\alpha$-Sasakian. When $\alpha=-2$, the structure is said to be Sasakian.

By [Friedrich and Ivanov 2002, Theorem 8.2], an almost contact metric manifold $\left(N^{2 n+1}, I, \xi, \eta, g\right)$ admits a connection $\nabla^{c}$ that preserves the almost contact metric 
structure and has totally skew-symmetric torsion tensor if and only if the Nijenhuis tensor of $I$, given by (1), is skew-symmetric and $\xi$ is a Killing vector field. This connection is unique.

In particular, on any quasi-Sasakian manifold $\left(N^{2 n+1}, I, \xi, \eta, g\right)$ there exists a unique connection $\nabla^{c}$ with totally skew-symmetric torsion, such that

$$
\nabla^{c} I=0, \quad \nabla^{c} g=0, \quad \nabla^{c} \eta=0 .
$$

Such a connection $\nabla^{c}$ is uniquely determined by

$$
g\left(\nabla_{X}^{c} Y, Z\right)=g\left(\nabla_{X}^{g} Y, Z\right)+\frac{1}{2}(d \eta \wedge \eta)(X, Y, Z),
$$

where $\nabla^{g}$ is the Levi-Civita connection and $\frac{1}{2}(d \eta \wedge \eta)$ is the torsion 3-form of $\nabla^{c}$.

Corollary 2.4. Let $\left(N^{2 n+1}, I, \xi, \eta, g\right)$ be a quasi-Sasakian $(2 n+1)$-manifold, and let $\Omega$ be a closed 2-form on $N^{2 n+1}$ that represents an integral cohomology class. Consider the circle bundle $S^{1} \hookrightarrow P \rightarrow N^{2 n+1}$ with connection 1-form $\theta$ whose curvature form is $d \theta=\pi^{*}(\Omega)$ for the projection $\pi: P \rightarrow N^{2 n+1}$. The almost Hermitian structure $(J, h)$ on $P$ defined by (2) and (4) is SKT if and only if $\Omega$ is I-invariant, $i_{\xi} \Omega=0$, and

$$
d \eta \wedge d \eta=-\Omega \wedge \Omega
$$

The Bismut connection $\nabla^{B}$ of $(J, h)$ on $P$ and the connection $\nabla^{c}$ on $N$ given by (10) are related by

$$
h\left(\nabla_{X}^{B} Y, Z\right)=\pi^{*} g\left(\nabla_{\pi_{*} X}^{c} \pi_{*} Y, \pi_{*} Z\right)
$$

for any vector fields $X, Y, Z \in \operatorname{Ker} \theta$.

Proof. Since $d \omega=0$, if we impose the SKT condition, then we get by using the previous theorem the equation (11).

The Bismut connection $\nabla^{B}$ associated to the Hermitian structure $(J, h)$ on $P$ is

$$
h\left(\nabla_{X}^{B} Y, Z\right)=h\left(\nabla_{X}^{h} Y, Z\right)-\frac{1}{2} d F(J X, J Y, J Z)
$$

for any vector fields $X, Y, Z$ on $P$, where $\nabla^{h}$ is the Levi-Civita connection associated to $h$. Then, for any $X, Y, Z$ in the kernel of $\theta$, we have

$$
h\left(\nabla_{X}^{B} Y, Z\right)=\pi^{*} g\left(\nabla_{X}^{h} Y, Z\right)+\frac{1}{2}\left(\pi^{*}(d \eta) \wedge \pi^{*} \eta\right)(X, Y, Z) .
$$

By [Ogawa 1963, Lemma 3] and the definition of $\nabla^{c}$, we get

$$
\begin{aligned}
h\left(\nabla_{X}^{B} Y, Z\right) & =\pi^{*} g\left(\nabla_{\pi_{*} X}^{g} \pi_{*} Y, \pi_{*} Z\right)+\frac{1}{2}\left(\pi^{*}(d \eta) \wedge \pi^{*} \eta\right)(X, Y, Z) \\
& =\pi^{*} g\left(\nabla_{\pi_{*}}^{c} \pi_{*} Y, \pi_{*} Z\right)
\end{aligned}
$$

for any $X, Y, Z$ in the kernel of $\theta$. 
Remark 2.5. If the structure $(I, \xi, \eta, g)$ is $\alpha$-Sasakian, equation (11) reads

$$
\Omega \wedge \Omega=-\alpha^{2} \omega \wedge \omega .
$$

In the case of a trivial $S^{1}$-bundle, that is, if we consider the natural almost Hermitian structure on the product $N^{2 n+1} \times \mathbb{R}$, we get this:

Corollary 2.6. Let $\left(N^{2 n+1}, I, \xi, \eta, g\right)$ be a $(2 n+1)$-dimensional almost contact metric manifold. Impose on the product $N^{2 n+1} \times \mathbb{R}$ the almost complex structure $J$ given by

$$
J X=I X \quad \text { for } X \in \operatorname{Ker} \eta \quad \text { and } \quad J \xi=-\frac{d}{d t},
$$

and the metric $h$ given by $h=g+(d t)^{2}$. The Hermitian structure $(J, h)$ is $S K T$ if and only if $(I, \xi, \eta, g)$ is normal, $d(I(d \omega))=d(d \eta \wedge \eta)$ and $d\left(I\left(i_{\xi} d \omega\right)\right)=0$, where $\omega$ denotes the fundamental 2-form of the almost contact metric structure $(g, I, \xi, \eta)$.

Corollary 2.7. Let $\left(N^{2 n+1}, I, \xi, \eta, g\right)$ be a $(2 n+1)$-dimensional quasi-Sasakian manifold with $d \eta \wedge d \eta=0$. The Hermitian structure $(J, h)$ on $N^{2 n+1} \times \mathbb{R}$ is $S K T$. Moreover, its Bismut connection $\nabla^{B}$ coincides with the unique connection $\nabla^{c}$ on $N^{2 n+1}$ given by (10).

Proof. In this case, since $d \omega=0$ we get $d(J d F)=-d(d \eta \wedge \eta)$. By using (12), we get $h\left(\nabla_{X}^{B} Y, Z\right)=g\left(\nabla_{X}^{c} Y, Z\right)$ for any vector fields $X, Y, Z$ on $N^{2 n+1}$.

2.1. Examples. We will present three examples of quasi-Sasakian Lie algebras satisfying the condition $d \eta \wedge d \eta=0$. By applying Corollary 2.7, one gets an SKT structure on the product of the corresponding simply connected Lie group by $\mathbb{R}$.

Example 2.8. Let $\mathfrak{s}$ be the 5-dimensional Lie algebra with structure equations

$$
\begin{aligned}
& d e^{1}=e^{13}+e^{23}+e^{25}-e^{34}+e^{35}, \\
& d e^{2}=2 e^{12}-2 e^{13}+e^{14}-e^{15}-e^{24}+e^{34}+e^{45}, \\
& d e^{3}=-e^{12}+e^{13}+e^{14}-e^{15}+2 e^{24}-2 e^{34}+e^{45}, \\
& d e^{4}=-e^{12}-e^{23}+e^{24}-e^{25}-e^{35}, \\
& d e^{5}=e^{12}-e^{13}-e^{24}+e^{34},
\end{aligned}
$$

where $e^{i j}=e^{i} \wedge e^{j}$. On $\mathfrak{s}$, take the quasi-Sasakian structure $(I, \xi, \eta, g)$ given by

$$
\eta=e^{5}, \quad I e^{1}=-e^{2}, \quad I e^{3}=-e^{4}, \quad \omega=-e^{12}-e^{34}, \quad g=\sum_{j=1}^{5}\left(e^{j}\right)^{2} .
$$

This quasi-Sasakian structure satisfies the condition $d(d \eta \wedge \eta)=0$. The Lie algebra $\mathfrak{s}$ is 2 -step solvable since the commutator

$$
\mathfrak{s}^{1}=[\mathfrak{s}, \mathfrak{s}]=\mathbb{R}\left\langle e_{1}-e_{4}, e_{2}+e_{3}, e_{1}-e_{2}+2 e_{3}-e_{5}\right\rangle
$$


is abelian, where $\left\{e_{1}, \ldots, e_{5}\right\}$ denotes the dual basis of $\left\{e^{1}, \ldots, e^{5}\right\}$. Moreover, $\mathfrak{s}$ has trivial center, is irreducible and nonunimodular, since the trace of $\operatorname{ad}_{e_{1}}$ is -3 .

Example 2.9. Consider the family of 2 -step solvable Lie algebras $\mathfrak{s}_{a}$ for $a \in \mathbb{R}-\{0\}$, given by

$$
\begin{aligned}
d e^{1}=a e^{23}+3 e^{25}, & d e^{3}=a e^{34}, \\
d e^{2}=-a e^{13}-3 e^{15}, & d e^{4}=0, \\
& d e^{5}=-\frac{1}{3} a^{2} e^{34} .
\end{aligned}
$$

The almost contact metric structure $(I, \xi, \eta, g)$ defined in (14) is quasi-Sasakian and satisfies the condition $d \eta \wedge d \eta=0$. The second cohomology group of $\mathfrak{s}_{a}$ is generated by $e^{12}$ and $e^{45}$.

Example 2.10. Another family of quasi-Sasakian Lie algebras that satisfies the condition $d \eta \wedge d \eta=0$ is $\mathfrak{g}_{b}$ for $b \in \mathbb{R}-\{0\}$, with structure equations

$$
\begin{array}{ll}
d e^{1}=b\left(e^{13}+e^{14}-e^{23}+e^{24}\right)+e^{25}, & d e^{3}=2 e^{45}, \\
d e^{2}=b\left(-e^{13}+e^{14}-e^{23}-e^{24}\right)-e^{15}, & d e^{4}=-2 e^{35}, \\
& d e^{5}=-4 b^{2} e^{34},
\end{array}
$$

and endowed with the quasi-Sasakian structure given by (14). The second cohomology group of $\mathfrak{g}_{b}$ is generated by $e^{12}$. The Lie algebras $\mathfrak{g}_{b}$ are not solvable since the commutators are $\left[\mathfrak{g}_{b}, \mathfrak{g}_{b}\right]=\mathfrak{g}_{b}$.

The Lie groups underlying Examples 2.9 and 2.10 also satisfy the conditions of Corollary 2.4 with $\Omega \wedge \Omega=0$, by just taking as connection 1-form the 1-form $e^{6}$ such that $d e^{6}=\lambda e^{12}$. Then, $\Omega=\lambda e^{12}$. With this expression of $d e^{6}$, we have

$$
d^{2} e^{6}=0, \quad J\left(d e^{6}\right)=d e^{6}, \quad \text { and } \quad d e^{6} \wedge d e^{6}=0 .
$$

Therefore, equation (11) is satisfied. Observe that $\lambda=0$ provides examples of trivial $S^{1}$-bundles.

The next example allows us to recover one of the 6-dimensional nilmanifolds found in [Fino et al. 2004]:

Example 2.11. Consider the 5-dimensional nilpotent Lie algebra with structure equations

$$
\begin{aligned}
& d e^{j}=0 \text { for } j=1, \ldots, 4, \\
& d e^{5}=e^{12}+e^{34},
\end{aligned}
$$

and endowed with the quasi-Sasakian structure given by (14). If we consider the closed 2-form $\Omega=e^{13}+e^{24}$ and apply Corollary 2.4, we see that there exists a nontrivial $S^{1}$-bundle over the corresponding 5-dimensional nilmanifold. Since $d e^{5} \wedge d e^{5}=-\Omega \wedge \Omega \neq 0$, the total space of this $S^{1}$-bundle is an SKT nilmanifold. More precisely, according to the classification given in [Fino et al. 2004] (see also 
[Ugarte 2007]), the nilmanifold is the one with underlying Lie algebra isomorphic to $\mathfrak{h}_{3} \oplus \mathfrak{h}_{3}$, where by $\mathfrak{h}_{3}$ we denote the real 3-dimensional Heisenberg Lie algebra.

Since the starting Lie algebra from Example 2.11 is Sasakian, it is natural to start with other 5-dimensional Sasakian Lie algebras to construct new SKT structures in dimension 6. A classification of 5-dimensional Sasakian Lie algebras was obtained in [Andrada et al. 2009].

Example 2.12. Consider the 5-dimensional Lie algebra $\mathfrak{k}_{3}$ with structure equations

$$
\begin{array}{ll}
d e^{1}=0, & d e^{4}=0, \\
d e^{2}=-e^{13}, & d e^{5}=\lambda e^{14}+\mu e^{23}, \\
d e^{3}=e^{12}, &
\end{array}
$$

where $\lambda, \mu<0$. By [Andrada et al. 2009], this algebra admits the Sasakian structure given by

$$
\begin{gathered}
I e^{1}=e^{4}, \quad I e^{2}=e^{3}, \quad \eta=e^{5}, \\
g=-\frac{1}{2} \lambda\left(e_{1}\right)^{2}-\frac{1}{2} \lambda\left(e_{2}\right)^{2}-\frac{1}{2} \mu\left(e_{3}\right)^{2}-\frac{1}{2} \mu\left(e_{4}\right)^{2}+\left(e_{5}\right)^{2},
\end{gathered}
$$

and is isomorphic to $\mathbb{R} \ltimes\left(\mathfrak{h}_{3} \times \mathbb{R}\right)$; moreover, the corresponding solvable simply connected Lie group admits a compact quotient by a discrete subgroup.

Consider on $\mathfrak{k}_{3}$ the closed 2-form $\Omega=\lambda e^{14}-\mu e^{23}$. The form $\Omega$ is $I$-invariant and satisfies $\Omega \wedge \Omega=-2 \lambda \mu e^{1234}$. Since $e^{5}$ is the contact form and $d e^{5} \wedge d e^{5}=2 \lambda \mu e^{1234}$, we get again by Corollary 2.4 an SKT structure on a nontrivial $S^{1}$-bundle over the 5-dimensional solvmanifold. We denote by $e^{6}$ the connection 1 -form.

The orthonormal basis $\left\{\alpha^{1}=e^{1}, \alpha^{2}=e^{4}, \alpha^{3}=e^{2}, \alpha^{4}=e^{3}, \alpha^{5}=e^{5}, \alpha^{6}=\theta\right\}$ for the SKT metric satisfies the equations

$$
\begin{array}{ll}
d \alpha^{1}=d \alpha^{2}=0, & d \alpha^{3}=-\alpha^{14}, \quad d \alpha^{4}=\alpha^{13}, \\
d \alpha^{5}=\lambda \alpha^{12}+\mu \alpha^{34}, & d \alpha^{6}=\lambda \alpha^{12}-\mu \alpha^{34},
\end{array}
$$

and the complex structure is given by $J\left(X_{1}\right)=X_{2}, J\left(X_{3}\right)=X_{4}$ and $J\left(X_{5}\right)=X_{6}$, where $\left\{X_{i}\right\}_{i=1}^{6}$ denotes the basis dual to $\left\{\alpha^{i}\right\}_{i=1}^{6}$. Since the fundamental 2-form is $F=\alpha^{12}+\alpha^{34}+\alpha^{56}$, the 3 -form torsion $T$ of the SKT structure is

$$
T=\lambda \alpha^{12}\left(\alpha^{5}+\alpha^{6}\right)+\mu \alpha^{34}\left(\alpha^{5}-\alpha^{6}\right) .
$$

Moreover, $* T=\lambda \alpha^{12}\left(\alpha^{5}+\alpha^{6}\right)-\mu \alpha^{34}\left(\alpha^{5}-\alpha^{6}\right)$, where $*$ denotes the metric's Hodge operator; this implies that the torsion form is also coclosed.

The only nonzero curvature forms $\left(\Omega^{B}\right)_{j}^{i}$ of the Bismut connection $\nabla^{B}$ are

$$
\left(\Omega^{B}\right)_{2}^{1}=-2 \lambda^{2} \alpha^{12} \text { and }\left(\Omega^{B}\right)_{4}^{3}=-2 \mu^{2} \alpha^{34} .
$$


A direct calculation shows that the 1-forms $\alpha^{5}$ and $\alpha^{6}$ and the 2-forms $\alpha^{12}$ and $\alpha^{34}$ are parallel with respect to the Bismut connection, which implies that $\nabla^{B} T=0$.

Finally, $\operatorname{Hol}\left(\nabla^{B}\right)=U(1) \times U(1) \subset U(3)$ since $\nabla^{B} \alpha^{i} \neq 0$ for $i=1,2,3,4$.

\section{SKT structures arising from Riemannian cones}

Let $N^{2 n+1}$ be a $(2 n+1)$-dimensional manifold endowed with an almost contact metric structure $(I, \xi, \eta, g)$, and denote by $\omega$ its fundamental 2-form.

The Riemannian cone of $N^{2 n+1}$ is defined as the manifold $N^{2 n+1} \times \mathbb{R}^{+}$equipped with the cone metric

$$
h=t^{2} g+(d t)^{2} .
$$

The cone $N^{2 n+1} \times \mathbb{R}^{+}$has a natural almost Hermitian structure defined by

$$
F=t^{2} \omega+t \eta \wedge d t .
$$

The almost complex structure $J$ on $N^{2 n+1} \times \mathbb{R}^{+}$defined by $(F, h)$ is given by

$$
J X=I X \quad \text { for } X \in \operatorname{Ker} \eta \quad \text { and } \quad J \xi=-t \frac{d}{d t} .
$$

In terms of a local orthonormal adapted coframe $\left\{e^{1}, \ldots, e^{2 n}\right\}$ for $g$ with

$$
\omega=-\sum_{j=1}^{n} e^{2 j-1} \wedge e^{2 j},
$$

we have

$$
\begin{aligned}
J e^{2 j-1} & =-e^{2 j}, \quad J e^{2 j}=e^{2 j-1} \quad \text { for } j=1, \ldots, n, \\
J\left(t e^{2 n+1}\right) & =d t, \quad J(d t)=-t e^{2 n+1} .
\end{aligned}
$$

The almost Hermitian structure $(J, h)$ on $N^{2 n+1} \times \mathbb{R}^{+}$is Kähler if and only if the almost contact metric structure $(I, \xi, \eta, g)$ on $N^{2 n+1}$ is Sasakian, that is, a normal contact metric structure.

If we impose that the almost Hermitian structure $(J, h)$ on $N^{2 n+1} \times \mathbb{R}^{+}$is SKT, we can prove the following:

Theorem 3.1. Consider a $(2 n+1)$-dimensional almost contact metric manifold $\left(N^{2 n+1}, I, \xi, \eta, g\right)$. The almost Hermitian structure $(J, h)$ given by $(15)$ and $(16)$ on the Riemannian cone $\left(N^{2 n+1} \times \mathbb{R}^{+}, h\right)$ is SKT if and only if $(I, \xi, \eta, g)$ is normal and

$$
-4 \eta \wedge \omega+2 I(d \omega)-2 d \eta \wedge \eta=d\left(I\left(i_{\xi} d \omega\right)\right),
$$

where $\omega$ denotes the fundamental 2-form of the almost contact metric structure $(I, \xi, \eta, g)$. 
Proof. $J$ is integrable if and only if the almost contact metric structure is normal. We compute $J d F$. We have

$$
\begin{aligned}
d F & =2 t d t \wedge \omega+t^{2} d \omega+t d \eta \wedge d t, \quad \text { and so } \\
J d F & =-2 t^{2} \eta \wedge \omega+t^{2} J(d \omega)-t^{2} d \eta \wedge \eta
\end{aligned}
$$

since $J \omega=\omega, J(d t)=-t \eta$ and $J d \eta=d \eta$. Moreover, with respect to an adapted basis $\left\{e^{1}, \ldots, e^{2 n+1}\right\}$ we can get, in a way similar to the proof of Theorem 2.3 , that

$$
J d \omega=I(d \omega)+I\left(i_{\xi} d \omega\right) \wedge J \eta .
$$

As a consequence, we get $J d F=-2 t^{2} \eta \wedge \omega+t^{2} I(d \omega)+t d t \wedge I\left(i_{\xi} d \omega\right)-t^{2} d \eta \wedge \eta$. Therefore, by imposing that $d(J d F)=0$, we obtain

$$
\begin{aligned}
-4 \eta \wedge \omega+2 I(d \omega)-2 d \eta \wedge \eta-d\left(I\left(i_{\xi} d \omega\right)\right) & =0, \\
-2 d(\eta \wedge \omega)+d(I(d \omega))-d(d \eta \wedge \eta) & =0 .
\end{aligned}
$$

Since the second equation is a consequence of the first, the Hermitian structure $(F, h)$ on the Riemannian cone $N^{2 n+1} \times \mathbb{R}^{+}$is SKT if and only if the almost contact metric structure $(I, \eta, \xi, g, \omega)$ on $N^{2 n+1}$ satisfies equation (19).

Remark 3.2. As a consequence of previous theorem, when $n=1$, equation (19) is satisfied if and only if the 3-dimensional manifold $N$ is Sasakian. On the other hand, if $n>1$ and the almost contact metric structure on $N^{2 n+1}$ is quasi-Sasakian (that is, $d \omega=0$ ), then the structure has to be Sasakian, that is, $d \eta=-2 \omega$.

Example 3.3. Consider the 5-dimensional Lie algebras $\mathfrak{g}_{a, b, c}$ with structure equations

$$
\begin{aligned}
& d e^{1}=a e^{23}+2 e^{25}+\left(-\frac{1}{2} a b+\frac{b^{3}}{2 a}+2 \frac{b}{a}\right) e^{34}+b e^{45}, \\
& d e^{2}=-a e^{13}-2 e^{15}-\frac{1}{2} b c e^{34}-b e^{35}, \\
& d e^{3}=\left(-\frac{4}{a}-\frac{b^{2}}{a}\right) e^{34}, \\
& d e^{4}=c e^{34}, \\
& d e^{5}=2 e^{12}+b e^{14}-b e^{23}+\left(2+b^{2}\right) e^{34},
\end{aligned}
$$

where $a, b, c \in \mathbb{R}$ and $a \neq 0$. They are endowed with the normal almost contact metric structure $(I, \xi, \eta, g, \omega)$ with

$$
I e^{1}=-e^{2}, \quad I e^{3}=-e^{4}, \quad \eta=e^{5}, \quad \omega=-e^{12}-e^{34} .
$$

This structure satisfies (19), and therefore the Riemannian cones over the corresponding simply connected Lie groups are SKT. 


\section{SKT SU(3)-structures}

Let $\left(M^{6}, J, h\right)$ be a 6-dimensional almost Hermitian manifold. An SU(3)-structure on $M^{6}$ is determined by the choice of a $(3,0)$-form $\Psi=\Psi_{+}+i \Psi_{-}$of unit norm. If $\Psi$ is closed, then the underlying almost complex structure $J$ is integrable and the manifold is Hermitian. We will denote the $\mathrm{SU}(3)$-structure $(J, h, \Psi)$ simply by $(F, \Psi)$, where $F$ is the fundamental 2-form, since from $F$ and $\Psi$ we can reconstruct the almost Hermitian structure.

Definition 4.1. We say that an $\mathrm{SU}(3)$-structure $(F, \Psi)$ on $M^{6}$ is $\mathrm{SKT}$ if

$$
d \Psi=0 \quad \text { and } \quad d(J d F)=0,
$$

where $J$ is the associated complex structure.

We will see the relation between SKT SU(3)-structures in dimension 6 and $\mathrm{SU}(2)$-structures in dimension 5.

First, we recall some facts about $\mathrm{SU}(2)$-structures on a 5-dimensional manifold. An SU(2)-structure on a 5-dimensional manifold $N^{5}$ is an SU(2)-reduction of the principal bundle of linear frames on $N^{5}$. By [Conti and Salamon 2007, Proposition 1], these structures are in one-to-one correspondence with quadruplets $\left(\eta, \omega_{1}, \omega_{2}, \omega_{3}\right)$, where $\eta$ is a 1 -form and $\omega_{i}$ are 2-forms on $N^{5}$ satisfying $\omega_{i} \wedge \omega_{j}=\delta_{i j} v$ and $v \wedge \eta \neq 0$ for some 4-form $v$, and $\omega_{2}(X, Y) \geq 0$ if $i_{X} \omega_{3}=i_{Y} \omega_{1}$, where $i_{X}$ denotes the contraction by $X$. Equivalently, an $\mathrm{SU}(2)$-structure on $N^{5}$ can be viewed as the datum of $\left(\eta, \omega_{1}, \Phi\right)$, where $\eta$ is a 1-form, $\omega_{1}$ is a 2 -form, and $\Phi=\omega_{2}+i \omega_{3}$ is a complex 2-form such that

$$
\eta \wedge \omega_{1} \wedge \omega_{1} \neq 0, \quad \Phi \wedge \Phi=0, \quad \omega_{1} \wedge \Phi=0, \quad \Phi \wedge \bar{\Phi}=2 \omega_{1} \wedge \omega_{1},
$$

and $\Phi$ is of type $(2,0)$ with respect to $\omega_{1}$.

Conti and Salamon [2007] locally characterize an SU(2)-structure as follows. If $\left(\eta, \omega_{1}, \omega_{2}, \omega_{3}\right)$ is an $\mathrm{SU}(2)$-structure on a 5-manifold $N^{5}$, then locally there exists an orthonormal basis of 1 -forms $\left\{e^{1}, \ldots, e^{5}\right\}$ such that

$$
\omega_{1}=e^{12}+e^{34}, \quad \omega_{2}=e^{13}-e^{24}, \quad \omega_{3}=e^{14}+e^{23}, \quad \eta=e^{5} .
$$

We can also consider the local tensor field $I$ given by

$$
I e^{1}=-e^{2}, \quad I e^{2}=e^{1}, \quad I e^{3}=-e^{4}, \quad I e^{4}=e^{3}, \quad I e^{5}=0 .
$$

This tensor gives rise to a global tensor field of type $(1,1)$ on the manifold $N^{5}$, defined by $\omega_{1}(X, Y)=g(X, I Y)$ for any vector fields $X$ and $Y$ on $N^{5}$, where $g$ is the Riemannian metric on $N^{5}$ underlying the SU(2)-structure. The tensor field $I$ satisfies $I^{2}=-\operatorname{Id}+\eta \otimes \xi$, where $\xi$ is the vector field on $N^{5}$ dual to the 1 -form $\eta$.

Therefore, given an $\mathrm{SU}(2)$-structure $\left(\eta, \omega_{1}, \omega_{2}, \omega_{3}\right)$ we also have an almost contact metric structure $(I, \xi, \eta, g)$ on the manifold, where $\omega_{1}$ is its fundamental form. 
Remark 4.2. Notice that we have two more almost contact metric structures when we consider $\omega_{2}$ and $\omega_{3}$ as fundamental forms.

If $N^{5}$ has an $\mathrm{SU}(2)$-structure $\left(\eta, \omega_{1}, \omega_{2}, \omega_{3}\right)$, the product $N^{5} \times \mathbb{R}$ has a natural $\mathrm{SU}(3)$-structure given by

$$
F=\omega_{1}+\eta \wedge d t \quad \text { and } \quad \Psi=\left(\omega_{2}+i \omega_{3}\right) \wedge(\eta-i d t) .
$$

By Corollary 2.6, the previous SU(3)-structure is SKT if and only if

$$
\begin{aligned}
d\left(I\left(d \omega_{1}\right)\right) & =d(d \eta \wedge \eta), & & d \omega_{2}=-3 \omega_{3} \wedge \eta, \\
d\left(I\left(i_{\xi} d \omega_{1}\right)\right) & =0, & & d \omega_{3}=3 \omega_{2} \wedge \eta,
\end{aligned}
$$

proving this:

Theorem 4.3. Suppose $N^{5}$ is a 5-dimensional manifold endowed with an $\mathrm{SU}(2)$ structure $\left(\eta, \omega_{1}, \omega_{2}, \omega_{3}\right)$. The $\mathrm{SU}(3)$-structure $(F, \Psi)$ given by (22) on the product $N^{5} \times \mathbb{R}$ is SKT if and only if the equations (23) are satisfied.

Example 4.4. On the 5-dimensional Lie algebras introduced in Examples 2.8, 2.9 and 2.10, consider the $\mathrm{SU}(2)$-structure given by

$$
\omega=\omega_{1}=e^{12}+e^{34}, \quad \omega_{2}=e^{13}-e^{24}, \quad \omega_{3}=e^{14}+e^{23} .
$$

For Example 2.8, we have

$$
d \omega_{2}=-2 \omega_{3} \wedge \eta-4\left(e^{124}-e^{134}\right) \quad \text { and } \quad d \omega_{3}=2 \omega_{2} \wedge \eta+4\left(e^{123}+e^{234}\right) .
$$

For Examples 2.9 and 2.10, we get $d \omega_{2}=-3 \omega_{3} \wedge \eta$ and $d \omega_{3}=3 \omega_{2} \wedge \eta$. Therefore one gets an SKT SU(3)-structure on the product of the corresponding simply connected Lie groups by $\mathbb{R}$.

We will study the existence of SKT SU(3)-structures on a Riemannian cone over a 5-dimensional manifold $N^{5}$ endowed with an $\mathrm{SU}(2)$-structure $\left(\eta, \omega_{1}, \omega_{2}, \omega_{3}\right)$. Then $N^{5}$ has an induced almost contact metric structure $(I, \xi, \eta, g)$, and $\omega_{1}$ is its fundamental form.

The Riemannian cone $\left(N^{5} \times \mathbb{R}^{+}, h\right)$ of $\left(N^{5}, g\right)$ has a natural SU(3)-structure defined by

$$
F=t^{2} \omega_{1}+t \eta \wedge d t \quad \text { and } \quad \Psi=t^{2}\left(\omega_{2}+i \omega_{3}\right) \wedge(t \eta-i d t) .
$$

In terms of a local orthonormal coframe $\left\{e^{1}, \ldots, e^{5}\right\}$ for $g$ such that

$$
\omega_{1}=-e^{12}-e^{34}, \quad \omega_{2}=-e^{13}+e^{24}, \quad \omega_{3}=-e^{14}-e^{23}, \quad \eta=e^{5},
$$

we have

$$
\begin{aligned}
& J e^{1}=-e^{2}, J e^{2}=e^{1}, \quad J e^{3}=-e^{4}, \\
& J e^{4}=e^{3}, \quad J\left(t e^{5}\right)=d t, \quad J(d t)=-t e^{5} .
\end{aligned}
$$


We recall that the $\mathrm{SU}(3)$-structure $(F, \Psi)$ on $N^{5} \times \mathbb{R}^{+}$is integrable if and only if the $\mathrm{SU}(2)$-structure $\left(\eta, \omega_{1}, \omega_{2}, \omega_{3}\right)$ on $N^{5}$ is Sasaki-Einstein, or equivalently if and only if

$$
d \eta=-2 \omega_{1}, \quad d \omega_{2}=-3 \omega_{3} \wedge \eta, \quad d \omega_{3}=3 \omega_{2} \wedge \eta .
$$

For the Riemannian cones, we can prove the following

Corollary 4.5. Let $N^{5}$ be a 5-dimensional manifold endowed with an SU(2)structure $\left(\eta, \omega_{1}, \omega_{2}, \omega_{3}\right)$. The $\mathrm{SU}(3)$-structure $(F, \Psi)$ on the Riemannian cone $\left(N^{5} \times \mathbb{R}^{+}, h\right)$ is SKT if and only if

$$
\begin{aligned}
-4 \eta \wedge \omega_{1}+2 I\left(d \omega_{1}\right)-2 d \eta \wedge \eta & =d\left(I\left(i_{\xi} d \omega_{1}\right)\right), \\
d \omega_{2} & =3 \omega_{3} \wedge \eta, \\
d \omega_{3} & =-3 \omega_{2} \wedge \eta .
\end{aligned}
$$

Proof. By imposing that $d \Psi=0$, we get the conditions $d \omega_{2}=-3 \omega_{3} \wedge \eta$ and $d \omega_{3}=3 \omega_{2} \wedge \eta$. By imposing $d(J d F)=0$, we get, as in the proof of Theorem 3.1, equation (19) for $\omega=\omega_{1}$.

\section{Almost contact metric structure induced on a hypersurface}

We study the almost contact metric structure induced naturally on any oriented hypersurface $N^{2 n+1}$ of a $(2 n+2)$-manifold $M^{2 n+2}$ equipped with an SKT structure.

Let $f: N^{2 n+1} \rightarrow M^{2 n+2}$ be an oriented hypersurface of a $(2 n+2)$-dimensional manifold $M^{2 n+2}$ endowed with an SKT structure $(J, h, F)$, and denote by $\mathbb{U}$ the unitary normal vector field. It is well known that $N^{2 n+1}$ inherits an almost contact metric structure $(I, \xi, \eta, g)$ such that $\eta$ and the fundamental 2-form $\omega$ are given by

$$
\eta=-f^{*}\left(i_{\Downarrow} F\right) \text { and } \omega=f^{*} F,
$$

where $F$ is the fundamental 2-form of the almost Hermitian structure; see, for instance, [Blair 2002].

Proposition 5.1. Suppose $f: N^{2 n+1} \rightarrow M^{2 n+2}$ is an immersion of an oriented $(2 n+1)$-dimensional manifold into a $(2 n+2)$-dimensional Hermitian manifold. If the Hermitian structure $(J, h)$ on $M^{2 n+2}$ is $S K T$, then the induced almost contact metric structure $(I, \xi, \eta, g)$ on $N^{2 n+1}$, with $\eta$ and $\omega$ given by (25), satisfies

$$
d\left(I d \omega-I\left(f^{*}\left(i_{\cup} d F\right)\right) \wedge \eta\right)=0 .
$$

Proof. Locally we can choose an adapted coframe $\left\{e^{1}, \ldots, e^{2 n+2}\right\}$ for the Hermitian structure so that the unitary normal vector field $\mathbb{U}$ is dual to $e^{2 n+2}$. Since the 
almost complex structure $J$ is given in this adapted basis by

$$
\begin{aligned}
J e^{2 j-1} & =-e^{2 j}, \quad J e^{2 j}=e^{2 j-1} \quad \text { for } j=1, \ldots, n, \\
J e^{2 n+1} & =e^{2 n+2}, \quad J e^{2 n+2}=-e^{2 n+1},
\end{aligned}
$$

it follows that the tensor field $I$ on $N^{2 n+1}$ has $I f^{*} e^{i}=f^{*} J e^{i}$ for $i=1, \ldots, 2 n+1$. That is,

$$
I f^{*} e^{2 j-1}=-f^{*} e^{2 j}, \quad I f^{*} e^{2 j}=f^{*} e^{2 j-1} \text { for } j=1, \ldots, n, \quad I f^{*} e^{2 n+1}=0 .
$$

However, $I f^{*} e^{2 n+2}=0 \neq f^{*} e^{2 n+1}=-f^{*} J e^{2 n+2}$.

We compute $f^{*} J d F$. First, we decompose (locally and in terms of the adapted basis) the differential of $F$ as

$$
d F=\alpha+\beta \wedge e^{2 n+1}+\gamma \wedge e^{2 n+2}+\mu \wedge e^{2 n+1} \wedge e^{2 n+2},
$$

where the local forms

$$
\alpha \in \bigwedge^{3}\left\langle e^{1}, \ldots, e^{2 n}\right\rangle, \quad \beta, \gamma \in \bigwedge^{2}\left\langle e^{1}, \ldots, e^{2 n}\right\rangle, \quad \mu \in \bigwedge^{1}\left\langle e^{1}, \ldots, e^{2 n}\right\rangle
$$

are generated only by $e^{1}, \ldots, e^{2 n}$. Then,

$$
J d F=J \alpha+J \beta \wedge e^{2 n+2}-J \gamma \wedge e^{2 n+1}+J \mu \wedge e^{2 n+1} \wedge e^{2 n+2} .
$$

Since $f^{*} e^{2 n+2}=0$ and $f^{*} e^{2 n+1}=\eta$, we have $f^{*} J d F=f^{*} J \alpha-\left(f^{*} J \gamma\right) \wedge \eta$. However, $f^{*}\left(i_{\cup} d F\right)=f^{*} \gamma+f^{*} \mu \wedge \eta$, which implies that

$$
I\left(f^{*}\left(i_{\cup} d F\right)\right)=I f^{*} \gamma=f^{*} J \gamma .
$$

On the other hand, $I d \omega=I d f^{*} F=I f^{*} d F=I f^{*} \alpha=f^{*} J \alpha$. We conclude that

$$
f^{*} J d F=f^{*} J \alpha-\left(f^{*} J \gamma\right) \wedge \eta=I d \omega-I\left(f^{*}\left(i_{\cup} d F\right)\right) \wedge \eta .
$$

Now, if the Hermitian structure is SKT, then $J d F$ is closed and the induced structure satisfies (26).

Remark 5.2. Notice that, using $i_{\bigcup} d F=\mathscr{L}_{\Downarrow} F-d i_{\Downarrow} F$, we can write (26) as

$$
d\left(I d \omega-I\left(f^{*}\left(\mathscr{L}_{\mathbb{U}} F\right)+d \eta\right) \wedge \eta\right)=0 .
$$

Therefore, if $f^{*}\left(\mathscr{L}_{\Downarrow} F\right)=0$, then the induced almost contact metric structure has to satisfy the equation $d(I d \omega-I(d \eta) \wedge \eta)=0$. In the case of the product $N^{2 n+1} \times \mathbb{R}$, the condition $f^{*}\left(\mathscr{L}_{\mathbb{U}} F\right)=0$ is satisfied. In the case of the Riemannian cone, we have $\mathscr{L}_{d / d t} F=2 t \omega+d t \wedge \eta$ and therefore $f^{*}\left(\mathscr{L}_{d / d t} F\right)=2 \omega$. In this way, we recover some of the equations obtained in Corollary 2.6 and Theorem 3.1.

Now we study the structure that is induced naturally on any oriented hypersurface $N^{5}$ of a 6-manifold $M^{6}$ equipped with an SKT SU(3)-structure. 
Let $f: N^{5} \rightarrow M^{6}$ be an oriented hypersurface of a 6-manifold $M^{6}$ endowed with an $\mathrm{SU}(3)$-structure $\left(F, \Psi=\Psi_{+}+i \Psi_{-}\right)$, and denote by $\mathbb{U}$ the unitary normal vector field. Then $N^{5}$ inherits an $\mathrm{SU}(2)$-structure $\left(\eta, \omega_{1}, \omega_{2}, \omega_{3}\right)$ given by

$$
\eta=-f^{*}\left(i_{\Downarrow} F\right), \quad \omega_{1}=f^{*} F, \quad \omega_{2}=-f^{*}\left(i_{\Perp} \Psi_{-}\right), \quad \omega_{3}=f^{*}\left(i_{\cup} \Psi_{+}\right) .
$$

Corollary 5.3. Let $f: N^{5} \rightarrow M^{6}$ be an immersion of an oriented 5-dimensional manifold into a 6-dimensional manifold with an $\mathrm{SU}(3)$-structure. If the $\mathrm{SU}(3)-$ structure is $S K T$, then the induced $\mathrm{SU}(2)$-structure on $N^{5}$ given by (27) satisfies

$$
\begin{aligned}
& d\left(I d \omega_{1}-I f^{*}\left(i_{\cup} d F\right) \wedge \eta\right)=0, \\
& d\left(\omega_{2} \wedge \eta\right)=0 \quad \text { and } \quad d\left(\omega_{3} \wedge \eta\right)=0 .
\end{aligned}
$$

Proof. Equation (28) follows from Proposition 5.1 by taking $\omega=\omega_{1}$. Locally, we can choose an adapted coframe $\left\{e^{1}, \ldots, e^{5}, e^{6}\right\}$ for the $\mathrm{SU}(3)$-structure such that the unitary normal vector field $\mathbb{U}$ is dual to $e^{6}$. From (27), it follows that $\omega_{2} \wedge \eta=f^{*} \Psi_{+}$and $\omega_{3} \wedge \eta=f^{*} \Psi_{-}$. If $\Psi=\Psi_{+}+i \Psi_{-}$is closed, then the induced structure satisfies (29).

5.1. A simple example. Consider the 6-dimensional nilmanifold $M^{6}$ whose underlying nilpotent Lie algebra has structure equations

$$
d e^{j}=0 \quad \text { for } j=1,2,3,6, \quad d e^{4}=e^{12}, \quad d e^{5}=e^{14},
$$

and is endowed with the SU(3)-structure given by

$$
F=-e^{14}-e^{26}-e^{53} \text { and } \Psi=\left(e^{1}-i e^{4}\right) \wedge\left(e^{2}-i e^{6}\right) \wedge\left(e^{5}-i e^{3}\right) .
$$

The oriented hypersurface with normal vector field dual to $e^{2}$ is a 5-dimensional nilmanifold $N^{5}$ that by [Conti and Salamon 2007] has no invariant hypo structures. However, the SU(2)-structure on $N^{5}$, namely,

$$
\eta=e^{2}, \quad \omega_{1}=-e^{14}-e^{53}, \quad \omega_{2}=-e^{15}-e^{34}, \quad \omega_{3}=-e^{13}-e^{45},
$$

satisfies (28) and (29). In Section 6, we will show that by using this SU(2)-structure and appropriate evolution equations, we can construct an SKT SU(3)-structure on the product of $N^{5}$ with an open interval.

\section{SKT evolution equations}

The goal here is to construct SKT SU(3)-structures by using appropriate evolution equations, starting from a suitable SU(2)-structure on a 5-dimensional manifold. We follow ideas of [Hitchin 2001] and [Conti and Salamon 2007].

Lemma 6.1. Let $\left(\eta(t), \omega_{1}(t), \omega_{2}(t), \omega_{3}(t)\right)$ be a family of $\mathrm{SU}(2)$-structures on a 5-dimensional manifold $N^{5}$ for $t \in(a, b)$. The $\mathrm{SU}(3)$-structure on $M^{6}=N^{5} \times(a, b)$ 
given by $F=\omega_{1}(t)+\eta(t) \wedge d t$ and $\Psi=\left(\omega_{2}(t)+i \omega_{3}(t)\right) \wedge(\eta(t)-i d t)$ satisfies the condition $d \Psi=0$ if and only if $\left(\eta(t), \omega_{1}(t), \omega_{2}(t), \omega_{3}(t)\right)$ is an $\mathrm{SU}(2)$-structure such that, for any $t$ in the open interval $(a, b)$,

$$
\begin{array}{ll}
\hat{d}\left(\omega_{2}(t) \wedge \eta(t)\right)=0, & \partial_{t}\left(\omega_{2}(t) \wedge \eta(t)\right)=-\hat{d} \omega_{3}(t), \\
\hat{d}\left(\omega_{3}(t) \wedge \eta(t)\right)=0, & \partial_{t}\left(\omega_{3}(t) \wedge \eta(t)\right)=\hat{d} \omega_{2}(t) .
\end{array}
$$

Here, $\hat{d}$ denotes the exterior differential on $N^{5}$ and $d$ is the exterior differential on $M^{6}$. We now present the additional evolution equations to be added to the last two of (31) in order to ensure that $d J d F=0$.

Proposition 6.2. Let $\left(\eta(t), \omega_{1}(t), \omega_{2}(t), \omega_{3}(t)\right)$ be a family of $\mathrm{SU}(2)$-structures on $N^{5}$ for $t \in(a, b)$. The $\mathrm{SU}(3)$-structure on $M^{6}=N^{5} \times(a, b)$ given by

$$
F=\omega_{1}(t)+\eta(t) \wedge d t \quad \text { and } \quad \Psi=\left(\omega_{2}(t)+i \omega_{3}(t)\right) \wedge(\eta(t)-i d t),
$$

has $J d F$ closed if and only if $\left(\eta(t), \omega_{1}(t), \omega_{2}(t), \omega_{3}(t)\right)$ satisfies the evolution equations

$$
\begin{aligned}
& \hat{d}\left(I_{t} \hat{d} \omega_{1}(t)-I_{t}\left(\partial_{t} \omega_{1}(t)+\hat{d} \eta(t)\right) \wedge \eta(t)\right)=0, \\
& \begin{aligned}
\partial_{t}\left(I_{t} \hat{d} \omega_{1}(t)\right. & \left.-I_{t}\left(\partial_{t} \omega_{1}(t)+\hat{d} \eta(t)\right) \wedge \eta(t)\right) \\
& =-\hat{d}\left(I_{t}\left(i_{\xi} \hat{d} \omega_{1}(t)\right)-I_{t}\left(i_{\xi}\left(\partial_{t} \omega_{1}(t)+\hat{d} \eta(t)\right)\right) \wedge \eta(t)\right),
\end{aligned}
\end{aligned}
$$

where $\xi(t)$ denotes the vector field on $N^{5}$ dual to $\eta(t)$ for each $t \in(a, b)$.

Proof. Since $F=\omega_{1}(t)+\eta(t) \wedge d t$, we have $d F=\hat{d} \omega_{1}+\left(\partial_{t} \omega_{1}+\hat{d} \eta\right) \wedge d t$. Define $\left\{e^{1}(t), \ldots, e^{4}(t), \eta(t)\right\}$ to be a local adapted basis for the $\mathrm{SU}(2)$-structure $\left(\eta(t), \omega_{1}(t), \omega_{2}(t), \omega_{3}(t)\right)$. Then, $\left\{e^{1}(t), \ldots, e^{4}(t), \eta(t), d t\right\}$ is an adapted basis for the $\mathrm{SU}(3)$-structure (32), and $J$ is given by

$$
\begin{array}{lll}
J e^{1}(t)=-e^{2}(t), & J e^{2}(t)=e^{1}(t), & J \eta(t)=d t, \\
J e^{3}(t)=-e^{4}(t), & J e^{4}(t)=e^{3}(t), & J d t=-\eta(t) .
\end{array}
$$

For each $t$, the structures $I_{t}$ induced on $N^{5}$ are given by

$$
\begin{aligned}
& I_{t} e^{1}(t)=-e^{2}(t), \quad I_{t} e^{2}(t)=e^{1}(t), \\
& I_{t} e^{3}(t)=-e^{4}(t), \quad I_{t} e^{4}(t)=e^{3}(t), \quad I_{t} \eta(t)=0 .
\end{aligned}
$$

Now, we can locally decompose a given $\tau(t) \in \Omega^{k}\left(N^{5}\right)$ for $t \in(a, b)$ as

$$
\tau(t)=\alpha(t)+\beta(t) \wedge \eta(t),
$$

where $\alpha(t) \in \bigwedge^{k}\left\langle e^{1}(t), \ldots, e^{4}(t)\right\rangle$ and $\beta(t) \in \bigwedge^{k-1}\left\langle e^{1}(t), \ldots, e^{4}(t)\right\rangle$. Therefore,

$$
\begin{aligned}
J \tau(t) & =J \alpha(t)+J \beta(t) \wedge J \eta(t)=I_{t} \alpha(t)+I_{t} \beta(t) \wedge d t \\
& =I_{t} \tau(t)-(-1)^{k} I_{t}\left(i_{\xi(t)} \tau(t)\right) \wedge d t .
\end{aligned}
$$


Applying this to $J d F$, we get

$$
\begin{aligned}
J d F= & J \hat{d} \omega_{1}-J\left(\partial_{t} \omega_{1}+\hat{d} \eta\right) \wedge \eta(t) \\
=I_{t} \hat{d} \omega_{1}-I_{t}\left(\partial_{t} \omega_{1}+\hat{d} \eta\right) & \wedge \eta(t)+I_{t}\left(i_{\xi(t)} \hat{d} \omega_{1}\right) \wedge d t \\
& -I_{t}\left(i_{\xi}\left(\partial_{t} \omega_{1}+\hat{d} \eta\right)\right) \wedge \eta(t) \wedge d t .
\end{aligned}
$$

Finally, taking the differential of $J d F$, we get

$$
\begin{aligned}
d J d F=\hat{d}\left(I_{t} \hat{d} \omega_{1}-I_{t}\left(\partial_{t} \omega_{1}+\hat{d} \eta\right) \wedge \eta(t)\right) & \\
+ & \partial_{t}\left(I_{t} \hat{d} \omega_{1}-I_{t}\left(\partial_{t} \omega_{1}+\hat{d} \eta\right) \wedge \eta(t)\right) \wedge d t \\
& +\hat{d}\left(I_{t}\left(i_{\xi(t)} \hat{d} \omega_{1}\right)-I_{t}\left(i_{\xi}\left(\partial_{t} \omega_{1}+\hat{d} \eta\right)\right) \wedge \eta(t)\right) \wedge d t .
\end{aligned}
$$

Remark 6.3. Observe that the first equation in (33) is exactly condition (28) for $F=\omega_{1}(t)+\eta(t) \wedge d t$. See Remark 5.2.

As a consequence of Lemma 6.1 and Proposition 6.2, we get the following:

Theorem 6.4. For $t \in(a, b)$, let $\left(\eta(t), \omega_{1}(t), \omega_{2}(t), \omega_{3}(t)\right)$ be a family of $\mathrm{SU}(2)$ structures on a 5-dimensional manifold $N^{5}$ such that

$$
\hat{d}\left(\omega_{2}(t) \wedge \eta(t)\right)=0 \quad \text { and } \quad \hat{d}\left(\omega_{3}(t) \wedge \eta(t)\right)=0
$$

for any $t$. If the evolution equations

$$
\begin{aligned}
& \hat{d}\left(I_{t} \hat{d} \omega_{1}(t)-I_{t}\left(\partial_{t} \omega_{1}(t)+\hat{d} \eta(t)\right) \wedge \eta(t)\right)=0, \\
& \begin{aligned}
\partial_{t}\left(I_{t} \hat{d} \omega_{1}(t)-I_{t}\left(\partial_{t} \omega_{1}(t)+\hat{d} \eta(t)\right) \wedge \eta(t)\right) \\
\quad=-\hat{d}\left(I_{t}\left(i_{\xi} \hat{d} \omega_{1}(t)\right)-I_{t}\left(i_{\xi}\left(\partial_{t} \omega_{1}(t)+\hat{d} \eta(t)\right)\right) \wedge \eta(t)\right), \\
\partial_{t}\left(\omega_{2}(t) \wedge \eta(t)\right)=-\hat{d} \omega_{3}(t), \\
\partial_{t}\left(\omega_{3}(t) \wedge \eta(t)\right)=\hat{d} \omega_{2}(t),
\end{aligned}
\end{aligned}
$$

are satisfied, then the $\mathrm{SU}(3)$-structure on $M=N \times(a, b)$ given by

$$
F=\omega_{1}(t)+\eta(t) \wedge d t \quad \text { and } \quad \Psi=\left(\omega_{2}(t)+i \omega_{3}(t)\right) \wedge(\eta(t)-i d t)
$$

is SKT.

Example 6.5. Consider the Lie algebra with structure equations

$$
d e^{j}=0 \quad \text { for }=1,2,3, \quad d e^{4}=e^{12}, \quad d e^{5}=e^{14},
$$

which underlies the 5-dimensional nilmanifold $N^{5}$ considered in Section 5.1. We endow it with the SU(2)-structure given by (30). It is easy to verify that

$$
d\left(\omega_{2} \wedge \eta\right)=d\left(\omega_{3} \wedge \eta\right)=d\left(\omega_{1} \wedge \omega_{1}\right)=0 .
$$


We evolve this $\mathrm{SU}(2)$-structure by

$$
\begin{aligned}
\omega_{1}(t) & =-e^{14}-e^{53}, & \omega_{2}(t) & =-\left(1+\frac{3}{2} t\right)^{1 / 3} e^{15}-\left(1+\frac{3}{2} t\right)^{-1 / 3} e^{34}, \\
\eta(t) & =\left(1+\frac{3}{2} t\right)^{1 / 3} e^{2}, & \omega_{3}(t) & =-\left(1+\frac{3}{2} t\right)^{1 / 3} e^{13}-\left(1+\frac{3}{2} t\right)^{-1 / 3} e^{45},
\end{aligned}
$$

where $t \in(-2 / 3, \infty)$.

For any $t \in(-2 / 3, \infty)$, the family $\left(\omega_{1}(t), \omega_{2}(t), \omega_{3}(t), \eta(t)\right)$ satisfies equations (34) and the last two equations of (35). Moreover, it satisfies the conditions

$$
\partial_{t} \omega_{1}(t)=0, \quad \hat{d}(\eta(t))=0, \quad i_{\xi}\left(\hat{d}\left(\omega_{1}(t)\right)\right)=0, \quad \partial_{t}\left(I_{t}\left(\hat{d} \omega_{1}(t)\right)\right)=0,
$$

which implies that the evolution equations (33) are also satisfied.

On the product $N^{5} \times \mathbb{R}$, we consider the local basis of 1 -forms

$$
\begin{array}{lll}
\beta^{1}=\left(1+\frac{3}{2} t\right)^{1 / 3} e^{1}, & \beta^{2}=\left(1+\frac{3}{2} t\right)^{-1 / 3} e^{4}, & \beta^{3}=e^{5}, \\
\beta^{4}=e^{3}, & \beta^{5}=\left(1+\frac{3}{2} t\right)^{1 / 3} e^{2}, & \beta^{6}=d t .
\end{array}
$$

The structure equations are

$$
\begin{array}{ll}
d \beta^{1}=-\frac{1}{2}\left(1+\frac{3}{2} t\right)^{-1} \beta^{16}, & d \beta^{4}=0, \\
d \beta^{2}=\left(1+\frac{3}{2} t\right)^{-1}\left(\beta^{15}+\frac{1}{2} \beta^{26}\right), & d \beta^{5}=-\frac{1}{2}\left(1+\frac{3}{2} t\right)^{-1} \beta^{56}, \\
d \beta^{3}=\beta^{12}, & d \beta^{6}=0 .
\end{array}
$$

Locally, $J$ is given by $J \beta^{1}=-\beta^{2}, J \beta^{3}=-\beta^{4}$ and $J \beta^{5}=\beta^{6}$. The fundamental form $F=-\beta^{12}-\beta^{34}+\beta^{56}$ satisfies $d(J d F)=0$, and the $(3,0)$-form $\Psi=$ $\left(\beta^{1}+i \beta^{2}\right) \wedge\left(\beta^{3}+i \beta^{4}\right) \wedge\left(\beta^{5}-i \beta^{6}\right)$ is closed. Therefore, $(F, \Psi)$ is a local SKT $\mathrm{SU}(3)$-structure on $N^{5} \times \mathbb{R}$.

Remark 6.6. A Hermitian structure $(J, h)$ on a 6-dimensional manifold $M^{6}$ is called balanced if $F \wedge F$ is closed, $F$ being the associated fundamental 2-form. The paper [Fernández et al. 2009] introduced the notion of balanced SU(2)-structures on 5-dimensional manifolds, together with appropriate evolution equations whose solution gives rise to a balanced $\mathrm{SU}(3)$-structure in six dimensions.

If $M^{6}$ is compact, then a balanced structure cannot be SKT; see, for instance, [Fino et al. 2004].

The SU(2)-structure (30) from the previous example is also balanced, and it gives rise to a balanced metric on the product of $N^{5}$ with a open interval; see [Fernández et al. 2009, (11)]. However, one can check directly that this solution is not SKT.

If $G$ is the nilpotent Lie group underlying $N^{5}$, the product $G \times \mathbb{R}$ has no leftinvariant SKT structures and does not admit any left-invariant complex structures; however, we can find a local SKT SU(3)-structure on it. 


\section{HKT structures}

We will now find conditions under which an $S^{1}$-bundle over a $(4 n+3)$-dimensional manifold endowed with three almost contact metric structures is hyper-Kähler with torsion (HKT, for short). Recall that a $4 n$-dimensional hyper-Hermitian manifold $\left(M^{4 n}, J_{1}, J_{2}, J_{3}, h\right)$ is a hypercomplex manifold $\left(M^{4 n}, J_{1}, J_{2}, J_{3}\right)$ endowed with a Riemannian metric $h$ compatible with the complex structures $J_{r}$ for $r=1,2,3$; that is, $h$ satisfies

$$
h\left(J_{r} X, J_{r} Y\right)=h(X, Y)
$$

for any $r=1,2,3$ and any vector fields $X$ and $Y$ on $M^{4 n}$.

A hyper-Hermitian manifold $\left(M^{4 n}, J_{1}, J_{2}, J_{3}, h\right)$ is called HKT if and only if

$$
J_{1} d F_{1}=J_{2} d F_{2}=J_{3} d F_{3},
$$

where $F_{r}$ denotes the fundamental 2-form associated to the Hermitian structure $\left(J_{r}, h\right)$; see [Grantcharov and Poon 2000].

We consider a $(4 n+3)$-dimensional manifold $N^{4 n+3}$ endowed with three almost contact metric structures $\left(I_{r}, \xi_{r}, \eta_{r}, g\right)$ for $r=1,2,3$, and satisfying

$$
\begin{aligned}
& I_{k}=I_{i} I_{j}-\eta_{j} \otimes \xi_{i}=-I_{j} I_{i}+\eta_{i} \otimes \xi_{j}, \\
& \xi_{k}=I_{i} \xi_{j}=-I_{j} \xi_{i}, \quad \eta_{k}=\eta_{i} I_{j}=-\eta_{j} I_{i} .
\end{aligned}
$$

By applying Theorem 2.3, we can construct hyper-Hermitian structures on $S^{1}$ bundles over $N^{4 n+3}$ and study when they are strong HKT.

Theorem 7.1. Let $N^{4 n+3}$ be a $(4 n+3)$-dimensional manifold with three normal almost contact metric structures $\left(I_{r}, \xi_{r}, \eta_{r}, g\right)$ for $r=1,2,3$, and satisfying (38). Let $\Omega$ be a closed 2-form on $N^{4 n+3}$ that represents an integral cohomology class, and that is $I_{r}$-invariant for every $r=1,2,3$. Consider the circle bundle $S^{1} \hookrightarrow$ $P \rightarrow N^{4 n+3}$ with a connection 1-form $\theta$ whose curvature form is $d \theta=\pi^{*}(\Omega)$, where $\pi: P \rightarrow N$ is the projection. The hyper-Hermitian structure $\left(J_{1}, J_{2}, J_{3}, h\right)$ on $P$ defined by (2) and (4) is HKT if and only if

$$
\begin{aligned}
\pi^{*}\left(I_{1}\left(d \omega_{1}\right)\right)-\pi^{*}\left(d \eta_{1}\right) \wedge \pi^{*} \eta_{1} & =\pi^{*}\left(I_{2}\left(d \omega_{2}\right)\right)-\pi^{*}\left(d \eta_{2}\right) \wedge \pi^{*} \eta_{2} \\
& =\pi^{*}\left(I_{3}\left(d \omega_{3}\right)\right)-\pi^{*}\left(d \eta_{3}\right) \wedge \pi^{*} \eta_{3}, \\
\pi^{*}\left(I_{1}\left(i_{\xi_{1}} d \omega_{1}\right)\right)=\pi^{*}\left(I_{2}\left(i_{\xi_{2}} d \omega_{2}\right)\right) & =\pi^{*}\left(I_{3}\left(i_{\xi_{3}} d \omega_{3}\right)\right),
\end{aligned}
$$

where $\omega_{r}$ is the fundamental form of the almost contact structure $\left(I_{r}, \xi_{r}, \eta_{r}, g\right)$. Moreover, the HKT structure is strong if and only if

$$
\begin{aligned}
d\left(\pi^{*}\left(I_{r}\left(i_{\xi_{r}} d \omega_{r}\right)\right)\right) & =0, \\
d\left(\pi^{*}\left(I_{r}\left(d \omega_{r}\right)-d \eta_{r} \wedge \eta_{r}\right)\right) & =\left(\pi^{*}\left(-I_{r}\left(i_{\xi_{r}} d \omega_{r}\right)\right)+\pi^{*} \Omega\right) \wedge \pi^{*} \Omega
\end{aligned}
$$

for every $r=1,2,3$. 
Proof. The almost hyper-Hermitian structure $\left(J_{1}, J_{2}, J_{3}, h\right)$ on $P$ defined by (2) and (4) is hyper-Hermitian if and only $\left(I_{r}, \xi_{r}, \eta_{r}, g\right)$ is normal and $d \theta$ is $J_{r}$-invariant for every $r=1,2,3$. The HKT condition is equivalent to (37). By (9), we have

$$
J_{r} d F_{r}=\pi^{*}\left(I_{r}\left(d \omega_{r}\right)\right)+\pi^{*}\left(I_{r}\left(i_{\xi_{r}} d \omega_{r}\right)\right) \wedge \theta-\pi^{*}\left(d \eta_{r}\right) \wedge \pi^{*} \eta_{r}-\theta \wedge d \theta,
$$

where $F_{r}$ is the fundamental 2-form of $\left(J_{r}, h\right)$. Therefore, condition (37) is satisfied if and only if (39) holds. Finally, the $J_{r} d F_{r}$ are closed if and only if (40) holds.

On $N^{4 n+3} \times \mathbb{R}$, consider the almost Hermitian structures $\left(J_{r}, F_{r}, h\right)$ defined by

$$
\begin{aligned}
h & =g+(d t)^{2}, & F_{r} & =\omega_{r}+\eta_{r} \wedge d t, \\
J_{r}\left(\eta_{r}\right) & =d t, & J_{r}(X) & =I_{r}(X) \quad \text { for } X \in \operatorname{Ker} \eta_{r} .
\end{aligned}
$$

By (38), we have

$$
\begin{aligned}
J_{1} J_{2}=J_{3}=-J_{2} J_{1}, \\
J_{1} \eta_{2}=I_{1} \eta_{2}=-\eta_{3}, \quad J_{2} \eta_{3}=I_{2} \eta_{3}=-\eta_{1}, \quad J_{3} \eta_{1}=I_{3} \eta_{1}=-\eta_{2} .
\end{aligned}
$$

Therefore, $\left(J_{r}, F_{r}, h\right)$ for $r=1,2,3$ is a hyper-Hermitian structure on $N^{4 n+3} \times \mathbb{R}$ if and only if the structures $\left(I_{r}, \xi_{r}, \eta_{r}, g\right)$ are normal.

Corollary 7.2. Let $N^{4 n+3}$ be a $(4 n+3)$-dimensional manifold endowed with three normal almost contact metric structures $\left(I_{r}, \xi_{r}, \eta_{r}, g\right)$ for $r=1,2,3$. On the product $N^{4 n+3} \times \mathbb{R}$, consider the hyper-Hermitian structure $\left(J_{1}, J_{2}, J_{3}, h\right)$ defined by (41). Then, $\left(J_{1}, J_{2}, J_{3}, h\right)$ is HKT if and only if

$$
\begin{aligned}
I_{1}\left(d \omega_{1}\right)-d \eta_{1} \wedge \eta_{1} & =I_{2}\left(d \omega_{2}\right)-d \eta_{2} \wedge \eta_{2}=I_{3}\left(d \omega_{3}\right)-d \eta_{3} \wedge \eta_{3}, \\
I_{1}\left(i_{\xi_{1}} d \omega_{1}\right) & =I_{2}\left(i_{\xi_{2}} d \omega_{2}\right)=I_{3}\left(i_{\xi_{3}} d \omega_{3}\right) .
\end{aligned}
$$

The HKT structure is strong if and only if

$$
d\left(I_{r}\left(i_{\xi_{r}} d \omega_{r}\right)\right)=0 \quad \text { and } \quad d\left(I_{r}\left(d \omega_{r}\right)-d \eta_{r} \wedge \eta_{r}\right)=0 \quad \text { for every } r=1,2,3 .
$$

Moreover, if $\left(J_{1}, J_{2}, J_{3}, h\right)$ is such that $d \eta_{1} \wedge \eta_{1}=d \eta_{2} \wedge \eta_{2}=d \eta_{3} \wedge \eta_{3}$ and one of the conditions

(a) $d \omega_{r}=0$ for any $r=1,2,3$, that is, $\left(I_{r}, \xi_{r}, \eta_{r}, g\right)$ is quasi-Sasakian for any $r=1,2,3$;or

(b) $d \omega_{i} \wedge \eta_{j} \wedge \eta_{k} \neq 0$, where $(i, j, k)$ is a permutation of $(1,2,3)$, as well as

$$
I_{1}\left(d \omega_{1}\right)=I_{2}\left(d \omega_{2}\right)=I_{3}\left(d \omega_{3}\right) \quad \text { and } \quad I_{1}\left(i_{\xi_{1}} d \omega_{1}\right)=I_{2}\left(i_{\xi_{2}} d \omega_{2}\right)=I_{3}\left(i_{\xi_{3}} d \omega_{3}\right) \text {, }
$$

is satisfied, then $\left(J_{1}, J_{2}, J_{3}, h\right)$ is HKT. In case (a), the HKT structure is strong. In case (b), the HKT structure is strong if and only if $d\left(I_{1}\left(d \omega_{1}\right)\right)=d\left(I_{1}\left(i_{\xi_{1}} d \omega_{1}\right)\right)=0$. 
Proof. By Theorem 7.1, the hyper-Hermitian structure $\left(J_{r}, F_{r}, h\right)$ for $r=1,2,3$ is HKT if and only if

$$
\begin{aligned}
I_{1}\left(d \omega_{1}\right)-d \eta_{1} \wedge \eta_{1} & =I_{2}\left(d \omega_{2}\right)-d \eta_{2} \wedge \eta_{2}=I_{3}\left(d \omega_{3}\right)-d \eta_{3} \wedge \eta_{3}, \\
I_{1}\left(i_{\xi_{1}} d \omega_{1}\right) & =I_{2}\left(i_{\xi_{2}} d \omega_{2}\right)=I_{3}\left(i_{\xi_{3}} d \omega_{3}\right) .
\end{aligned}
$$

Locally, we write

$$
d \omega_{r}=\alpha_{r}+\sum_{i=1}^{3} \beta_{i}^{r} \wedge \eta_{i}+\sum_{i<j=1}^{3} \gamma_{i j}^{r} \wedge \eta_{i} \wedge \eta_{j}+\rho_{r} \eta_{1} \wedge \eta_{2} \wedge \eta_{3}
$$

where $\rho_{r}$ are smooth functions, while $\alpha_{r}, \beta_{i}^{r}$, and $\gamma_{i j}^{r}$ are respectively 3 -forms, 2-forms, and 1-forms in $\bigcap_{i=1}^{3} \operatorname{Ker} \eta_{i}$.

By first using the normality of the three almost contact metric structures, and then that $i_{\xi_{r}} d \eta_{r}=0$ and $I_{r}\left(d \eta_{r}\right)=d \eta_{r}$, locally we can write

$$
\begin{aligned}
& d \eta_{1}=A_{1}+B_{1} \wedge \eta_{2}-I_{1} B_{1} \wedge \eta_{3}+C_{1} \eta_{2} \wedge \eta_{3}, \\
& d \eta_{2}=A_{2}+B_{2} \wedge \eta_{1}+I_{2} B_{2} \wedge \eta_{3}+C_{2} \eta_{1} \wedge \eta_{3}, \\
& d \eta_{3}=A_{3}+B_{3} \wedge \eta_{1}-I_{3} B_{3} \wedge \eta_{2}+C_{3} \eta_{1} \wedge \eta_{2},
\end{aligned}
$$

where $I_{r} A_{r}=A_{r}$. Here, the $A_{r}$ and $B_{r}$ are respectively 2-forms and 1-forms in $\bigcap_{i=1}^{3} \operatorname{Ker} \eta_{i}$, while the $C_{r}$ are smooth functions. We have

$$
J_{r}\left(d F_{r}\right)=J_{r}\left(d \omega_{r}\right)+J_{r}\left(d \eta_{r} \wedge d t\right)=J_{r}\left(d \omega_{r}\right)-d \eta_{r} \wedge \eta_{r}
$$

Therefore, by using (43) and (44), we obtain

$$
\begin{aligned}
J_{1}\left(d F_{1}\right)= & I_{1} \alpha_{1}+I_{1} \beta_{1}^{1} \wedge d t-A_{1} \wedge \eta_{1}-I_{1} \beta_{3}^{1} \wedge \eta_{2}-I_{1} \beta_{2}^{1} \wedge \eta_{3} \\
& -I_{1} \gamma_{13}^{1} \wedge \eta_{2} \wedge d t+I_{1} \gamma_{12}^{1} \wedge \eta_{3} \wedge d t+B_{1} \wedge \eta_{1} \wedge \eta_{2}-I_{1} B_{1} \wedge \eta_{1} \wedge \eta_{3} \\
& +I_{1} \gamma_{23}^{1} \wedge \eta_{2} \wedge \eta_{3}+\rho_{1} \eta_{2} \wedge \eta_{3} \wedge d t-C_{1} \eta_{1} \wedge \eta_{2} \wedge \eta_{3}, \\
J_{2}\left(d F_{2}\right)= & I_{2} \alpha_{2}+I_{2} \beta_{2}^{2} \wedge d t-I_{2} \beta_{3}^{2} \wedge \eta_{1}-A_{2} \wedge \eta_{2}+I_{2} \beta_{1}^{2} \wedge \eta_{3} \\
& +I_{2} \gamma_{23}^{2} \wedge \eta_{1} \wedge d t+I_{2} \gamma_{12}^{2} \wedge \eta_{3} \wedge d t-B_{2} \wedge \eta_{1} \wedge \eta_{2}+I_{2} \gamma_{13}^{2} \wedge \eta_{1} \wedge \eta_{3} \\
& +I_{2} B_{2} \wedge \eta_{2} \wedge \eta_{3}-\rho_{2} \eta_{1} \wedge \eta_{3} \wedge d t+C_{2} \eta_{1} \wedge \eta_{2} \wedge \eta_{3}, \\
J_{3}\left(d F_{3}\right)= & I_{3} \alpha_{3}+I_{3} \beta_{3}^{3} \wedge d t+I_{3} \beta_{2}^{3} \wedge \eta_{1}-I_{3} \beta_{1}^{3} \wedge \eta_{2}-A_{3} \wedge \eta_{3} \\
& +I_{3} \gamma_{23}^{3} \wedge \eta_{1} \wedge d t-I_{3} \gamma_{13}^{3} \wedge \eta_{2} \wedge d t+I_{3} \gamma_{12}^{3} \wedge \eta_{1} \wedge \eta_{2}-B_{3} \wedge \eta_{1} \wedge \eta_{3} \\
& +I_{3} B_{3} \wedge \eta_{2} \wedge \eta_{3}+\rho_{3} \eta_{1} \wedge \eta_{2} \wedge d t-C_{3} \eta_{1} \wedge \eta_{2} \wedge \eta_{3} .
\end{aligned}
$$


The conditions (42) are satisfied if and only if

$$
\begin{aligned}
\gamma_{12}^{1} & =\gamma_{13}^{1}=\gamma_{12}^{2}=\gamma_{23}^{2}=\gamma_{13}^{3}=\gamma_{23}^{3}=0, \\
\rho_{r} & =0, \quad C_{1}=-C_{2}=C_{3}, \\
I_{1} \alpha_{1} & =I_{2} \alpha_{2}=I_{3} \alpha_{3}, \quad I_{1} \beta_{1}^{1}=I_{2} \beta_{2}^{2}=I_{3} \beta_{3}^{3}, \\
A_{1} & =I_{2} \beta_{3}^{2}=-I_{3} \beta_{2}^{3}, \quad A_{2}=-I_{1} \beta_{3}^{1}=I_{3} \beta_{1}^{3}, \quad A_{3}=I_{1} \beta_{2}^{1}=-I_{2} \beta_{1}^{2}, \\
B_{1} & =-B_{2}=I_{3} \gamma_{12}^{3}, \quad-I_{1} B_{1}=-B_{3}=I_{2} \gamma_{13}^{2}, \quad I_{2} B_{2}=I_{3} B_{3}=I_{1} \gamma_{23}^{1} .
\end{aligned}
$$

Since $I_{r} A_{r}=A_{r}$ the coefficients $\beta_{i}^{r}$ for $r \neq i=1,2,3$ must satisfy the conditions

$$
I_{i}\left(\beta_{j}^{i}-I_{k} \beta_{j}^{i}\right)=0 \quad \text { for all } i, j, k=1,2,3 \text { with } i \neq j, j \neq k \text { and } k \neq i .
$$

The last three equations in (45) are satisfied if and only if $\gamma_{23}^{1}=\gamma_{13}^{2}=\gamma_{12}^{3}=0$.

Thus, finally, we obtain

$$
\begin{aligned}
d \omega_{r} & =\alpha_{r}+\sum_{i=1}^{3} \beta_{i}^{r} \wedge \eta_{i}, \quad d \eta_{i}=A_{i}+\lambda \eta_{j} \wedge \eta_{k}, \\
0 & =I_{i}\left(\beta_{j}^{i}-I_{k} \beta_{j}^{i}\right) \quad \text { for all } i, j, k=1,2,3 \text { with } i \neq j, j \neq k \text { and } k \neq i, \\
I_{1} \alpha_{1} & =I_{2} \alpha_{2}=I_{3} \alpha_{3}, \\
A_{1} & =I_{2} \beta_{3}^{2}=-I_{3} \beta_{2}^{3}, \quad A_{2}=-I_{1} \beta_{3}^{1}=I_{3} \beta_{1}^{3}, \quad A_{3}=I_{1} \beta_{2}^{1}=-I_{2} \beta_{1}^{2}
\end{aligned}
$$

for any even permutation of $(1,2,3)$.

The expression for $d\left(J_{1} d F_{1}\right)$ is

$$
\begin{aligned}
d\left(J_{1} d F_{1}\right) & =d\left(I_{1}\left(d \omega_{1}\right)+I_{1}\left(i_{\xi_{1}} d \omega_{1}\right) \wedge d t\right)-d\left(\left(d \eta_{1}\right) \wedge \eta_{1}\right) \\
& =d\left(I_{1}\left(d \omega_{1}\right)\right)+d\left(I_{1}\left(i_{\xi_{1}} d \omega_{1}\right)\right) \wedge d t-d \eta_{1} \wedge d \eta_{1} \\
& =d\left(I_{1}\left(d \omega_{1}\right)-d \eta_{1} \wedge \eta_{1}\right)+d\left(I_{1}\left(i_{\xi_{1}} d \omega_{1}\right)\right) \wedge d t
\end{aligned}
$$

and thus the HKT structure is strong if and only if

$$
d\left(I_{1}\left(d \omega_{1}\right)-d \eta_{1} \wedge \eta_{1}\right)=0 \quad \text { and } \quad d\left(I_{1}\left(i_{\xi_{1}} d \omega_{1}\right)\right)=0 .
$$

To prove the last part of the corollary it suffices to consider coefficients $\beta_{r}^{i}=0$ if $r \neq i$ in expression (43).

Example 7.3. Consider the 7-dimensional Lie group $G=\mathrm{SU}(2) \ltimes \mathbb{R}^{4}$, with structure equations

$$
\begin{array}{rlrl}
d e^{1} & =-\frac{1}{2} e^{25}-\frac{1}{2} e^{36}-\frac{1}{2} e^{47}, & & d e^{5}=e^{67}, \\
d e^{2}=\frac{1}{2} e^{15}+\frac{1}{2} e^{37}-\frac{1}{2} e^{46}, & & d e^{6}=-e^{57}, \\
d e^{3} & =\frac{1}{2} e^{16}-\frac{1}{2} e^{27}+\frac{1}{2} e^{45}, & & d e^{7}=e^{56} . \\
d e^{4} & =\frac{1}{2} e^{17}+\frac{1}{2} e^{26}-\frac{1}{2} e^{35}, & &
\end{array}
$$


By [Fino and Tomassini 2008], $G$ admits a compact quotient $M^{7}=\Gamma \backslash G$ by a uniform discrete subgroup $\Gamma$, and is endowed with a weakly generalized $G_{2}$-structure. By [Barberis and Fino 2008], $M^{7} \times S^{1}$ admits a strong HKT structure. We can show that $M^{7}$ has three normal almost contact metric structures $\left(I_{r}, \xi_{r}, \eta_{r}, g\right)$ for $r=1,2,3$ that are given by

$$
\begin{array}{llll}
I_{1} e^{1}=e^{2}, & I_{1} e^{3}=e^{4}, & I_{1} e^{5}=e^{6}, & \eta_{1}=e^{7}, \\
I_{2} e^{1}=e^{3}, & I_{2} e^{2}=-e^{4}, & I_{2} e^{5}=-e^{7}, & \eta_{2}=e^{6}, \\
I_{3} e^{1}=e^{4}, & I_{3} e^{2}=e^{3}, & I_{3} e^{6}=e^{7}, & \eta_{3}=e^{5}
\end{array}
$$

and that satisfy the conditions of Corollary 7.2(a).

\section{References}

[Andrada et al. 2009] A. Andrada, A. Fino, and L. Vezzoni, "A class of Sasakian 5-manifolds", Transformation Groups 14:3 (2009), 493-512. MR 2534796 Zbl 1185.53046 arXiv 0807.1800

[Barberis and Fino 2008] M. L. Barberis and A. Fino, "New HKT manifolds arising from quaternionic representations", preprint, 2008. To appear in Math. Z. arXiv 0805.2335

[Barberis et al. 2009] M. L. Barberis, I. G. Dotti, and M. Verbitsky, "Canonical bundles of complex nilmanifolds, with applications to hypercomplex geometry", Math. Res. Lett. 16:2 (2009), 331-347. MR 2010g:53083 Zbl 1178.32014

[Bismut 1989] J.-M. Bismut, "A local index theorem for non-Kähler manifolds", Math. Ann. 284:4 (1989), 681-699. MR 91i:58140

[Blair 1967] D. E. Blair, "The theory of quasi-Sasakian structures", J. Differential Geometry 1 (1967), 331-345. MR 37 \#2127 Zbl 0163.43903

[Blair 2002] D. E. Blair, Riemannian geometry of contact and symplectic manifolds, Progress in Mathematics 203, Birkhäuser, Boston, MA, 2002. MR 2002m:53120 Zbl 1011.53001

[Boyer and Galicki 1999] C. Boyer and K. Galicki, "3-Sasakian manifolds", pp. 123-184 in Surveys in differential geometry: Essays on Einstein manifolds, edited by C. LeBrun and M. Wang, Surv. Differ. Geom. 6, Int. Press, Boston, 1999. MR 2001m:53076 Zbl 1008.53047

[Conti and Salamon 2007] D. Conti and S. Salamon, "Generalized Killing spinors in dimension 5", Trans. Amer. Math. Soc. 359:11 (2007), 5319-5343. MR 2008h:53077 Zbl 1130.53033

[Egidi 2001] N. Egidi, "Special metrics on compact complex manifolds", Differential Geom. Appl. 14:3 (2001), 217-234. MR 2002b:32038 Zbl 0981.32014

[Fernández et al. 2009] M. Fernández, A. Tomassini, L. Ugarte, and R. Villacampa, "Balanced Hermitian metrics from SU(2)-structures", J. Math. Phys. 50:3 (2009), 033507, 15. MR 2010f:53124 Zbl 05646541

[Fino and Grantcharov 2004] A. Fino and G. Grantcharov, "Properties of manifolds with skewsymmetric torsion and special holonomy", Adv. Math. 189:2 (2004), 439-450. MR 2005k:53062 Zbl 1114.53043

[Fino and Tomassini 2008] A. Fino and A. Tomassini, "Generalized $G_{2}$-manifolds and SU(3)structures", Internat. J. Math. 19:10 (2008), 1147-1165. MR 2009m:53054 Zbl 1168.53012

[Fino and Tomassini 2009] A. Fino and A. Tomassini, "Blow-ups and resolutions of strong Kähler with torsion metrics", Adv. Math. 221:3 (2009), 914-935. MR 2010d:32012 
[Fino et al. 2004] A. Fino, M. Parton, and S. Salamon, "Families of strong KT structures in six dimensions", Comment. Math. Helv. 79:2 (2004), 317-340. MR 2005f:53061 Zbl 1062.53062

[Friedrich and Ivanov 2002] T. Friedrich and S. Ivanov, "Parallel spinors and connections with skew-symmetric torsion in string theory", Asian J. Math. 6:2 (2002), 303-335. MR 2003m:53070 Zbl 1127.53304

[Gates et al. 1984] S. J. Gates, Jr., C. M. Hull, and M. Roček, "Twisted multiplets and new supersymmetric nonlinear $\sigma$-models", Nuclear Phys. B 248:1 (1984), 157-186. MR 87b:81108

[Gauduchon 1984] P. Gauduchon, "La 1-forme de torsion d'une variété hermitienne compacte", Math. Ann. 267:4 (1984), 495-518. MR 87a:53101 Zbl 0523.53059

[Grantcharov and Poon 2000] G. Grantcharov and Y. S. Poon, "Geometry of hyper-Kähler connections with torsion", Comm. Math. Phys. 213:1 (2000), 19-37. MR 2002a:53059

[Grantcharov et al. 2008] D. Grantcharov, G. Grantcharov, and Y. S. Poon, "Calabi-Yau connections with torsion on toric bundles", J. Differential Geom. 78:1 (2008), 13-32. MR 2009g:32052 Zbl 1171.53044

[Hitchin 2001] N. Hitchin, "Stable forms and special metrics", pp. 70-89 in Global differential geometry: The mathematical legacy of Alfred Gray (Bilbao, 2000), edited by M. Fernández and J. A. Wolf, Contemp. Math. 288, Amer. Math. Soc., Providence, RI, 2001. MR 2003f:53065 Zbl 1004.53034

[Howe and Papadopoulos 1996] P. S. Howe and G. Papadopoulos, "Twistor spaces for hyper-Kähler manifolds with torsion”, Phys. Lett. B 379:1-4 (1996), 80-86. MR 97h:53073

[Ivanov and Papadopoulos 2001] S. Ivanov and G. Papadopoulos, "Vanishing theorems and string backgrounds", Classical Quantum Gravity 18:6 (2001), 1089-1110. MR 2002h:53076 Zbl 0990. 53078

[Kobayashi 1956] S. Kobayashi, "Principal fibre bundles with the 1-dimensional toroidal group", Tôhoku Math. J. (2) 8 (1956), 29-45. MR 18,328a Zbl 0075.32103

[Ogawa 1963] Y. Ogawa, "Some properties on manifolds with almost contact structures", Tôhoku Math. J. (2) 15 (1963), 148-161. MR 27 \#704 Zbl 0147.41002

[Sasaki and Hatakeyama 1961] S. Sasaki and Y. Hatakeyama, "On differentiable manifolds with certain structures which are closely related to almost contact structure, II", Tôhoku Math. J. (2) 13 (1961), 281-294. MR 25 \#1513 Zbl 0112.14002

[Strominger 1986] A. Strominger, "Superstrings with torsion”, Nuclear Phys. B 274:2 (1986), 253 284. MR $87 \mathrm{~m}: 81177$

[Swann 2010] A. Swann, "Twisting Hermitian and hypercomplex geometries”, Duke Math. J. 155 (2010), 403-431.

[Ugarte 2007] L. Ugarte, "Hermitian structures on six-dimensional nilmanifolds", Transformation Groups 12:1 (2007), 175-202. MR 2008e:53139 Zbl 1129.53052

Received September 23, 2009.

\section{MARISA FERNÁNDEZ}

UNIVERSIDAD DEL PAÍS VASCO

DePaRtamento de Matemáticas

Facultad de Ciencia y TeCnOlogía

APARTADO 644

48080 BILBAO

SPAIN

marisa.fernandez@ehu.es 
ANNA FINO

UNIVERSITÀ DI TORINO

DiPARTIMENTO DI MATEMATICA

Via Carlo Alberto 10

I-10123 TORINO

ITALY

annamaria.fino@unito.it

LUIS UGARTE

UNIVERSIDAD DE ZARAGOZA

DEPARTAMENTO DE MATEMÁTICAS-IUMA

CAMPus Plaza San Francisco

50009 ZARAGOZA

SPAIN

ugarte@unizar.es

RAQUEL VILLACAMPA

Centro Universitario de la Defensa

ACademia General Militar

CRTA. DE HUESCA S/N

50090 ZARAGOZA

SPAIN

raquelvg@unizar.es 


\title{
PACIFIC JOURNAL OF MATHEMATICS
}

\author{
http://www.pjmath.org \\ Founded in 1951 by \\ E. F. Beckenbach (1906-1982) and F. Wolf (1904-1989)
}

\section{EDITORS}

V. S. Varadarajan (Managing Editor)

Department of Mathematics

University of California

Los Angeles, CA 90095-1555

pacific@math.ucla.edu

Vyjayanthi Chari

Department of Mathematics

University of California

Riverside, CA 92521-0135

chari@math.ucr.edu

Robert Finn

Department of Mathematics Stanford University

Stanford, CA 94305-2125

finn@math.stanford.edu

Kefeng Liu

Department of Mathematics

University of California

Los Angeles, CA 90095-1555

liu@math.ucla.edu
Darren Long

Department of Mathematics

University of California

Santa Barbara, CA 93106-3080

long@math.ucsb.edu

Jiang-Hua Lu

Department of Mathematics

The University of Hong Kong

Pokfulam Rd., Hong Kong jhlu@maths.hku.hk

Alexander Merkurjev

Department of Mathematics

University of California

Los Angeles, CA 90095-1555

merkurev@math.ucla.edu
Sorin Popa

Department of Mathematics University of California

Los Angeles, CA 90095-1555 popa@math.ucla.edu

Jie Qing

Department of Mathematics

University of California

Santa Cruz, CA 95064

qing@cats.ucsc.edu

Jonathan Rogawski

Department of Mathematics

University of California

Los Angeles, CA 90095-1555

jonr@math.ucla.edu

\section{PRODUCTION}

pacific@math.berkeley.edu

\begin{abstract}
Silvio Levy, Scientific Editor Matthew Cargo, Senior Production Editor
\end{abstract}
ACADEMIA SINICA, TAIPEI

CALIFORNIA INST. OF TECHNOLOGY

INST. DE MATEMÁTICA PURA E APLICADA

KEIO UNIVERSITY

MATH. SCIENCES RESEARCH INSTITUTE

NEW MEXICO STATE UNIV.

OREGON STATE UNIV.

\section{SUPPORTING INSTITUTIONS}

STANFORD UNIVERSITY
UNIV. OF BRITISH COLUMBIA
UNIV. OF CALIFORNIA, BERKELEY
UNIV. OF CALIFORNIA, DAVIS
UNIV. OF CALIFORNIA, LOS ANGELES
UNIV. OF CALIFORNIA, RIVERSIDE
UNIV. OF CALIFORNIA, SAN DIEGO
UNIV. OF CALIF., SANTA BARBARA

UNIV. OF CALIF., SANTA CRUZ

UNIV. OF MONTANA

UNIV. OF OREGON

UNIV. OF SOUTHERN CALIFORNIA

UNIV. OF UTAH

UNIV. OF WASHINGTON

WASHINGTON STATE UNIVERSITY

These supporting institutions contribute to the cost of publication of this Journal, but they are not owners or publishers and have no responsibility for its contents or policies.

See inside back cover or www.pjmath.org for submission instructions.

The subscription price for 2011 is US \$420/year for the electronic version, and \$485/year for print and electronic.

Subscriptions, requests for back issues from the last three years and changes of subscribers address should be sent to Pacific Journal of Mathematics, P.O. Box 4163, Berkeley, CA 94704-0163, U.S.A. Prior back issues are obtainable from Periodicals Service Company, 11 Main Street, Germantown, NY 12526-5635. The Pacific Journal of Mathematics is indexed by Mathematical Reviews, Zentralblatt MATH, PASCAL CNRS Index, Referativnyi Zhurnal, Current Mathematical Publications and the Science Citation Index.

The Pacific Journal of Mathematics (ISSN 0030-8730) at the University of California, c/o Department of Mathematics, 969 Evans Hall, Berkeley, CA 94720-3840, is published monthly except July and August. Periodical rate postage paid at Berkeley, CA 94704, and additional mailing offices. POSTMASTER: send address changes to Pacific Journal of Mathematics, P.O. Box 4163, Berkeley, CA 94704-0163.

PJM peer review and production are managed by EditFLOW ${ }^{\mathrm{TM}}$ from Mathematical Sciences Publishers.

PUBLISHED BY PACIFIC JOURNAL OF MATHEMATICS

at the University of California, Berkeley 94720-3840

A NON-PROFIT CORPORATION

Typeset in LATEX

Copyright $(02011$ by Pacific Journal of Mathematics 


\section{PACIFIC JOURNAL OF MATHEMATICS}

Volume $249 \quad$ No. $1 \quad$ January 2011

Metabelian SL $(n, \mathbb{C})$ representations of knot groups, II: Fixed points

HANS U. BODEN and STEFAN FRIEDL

Lewis-Zagier correspondence for higher-order forms

ANTON DEITMAR

Topology of positively curved 8-dimensional manifolds with symmetry

ANAND DESSAI

Strong Kähler with torsion structures from almost contact manifolds

MARISA FERnÁNDEZ, ANNA FINO, LUIS UGARTE and RAQUEL

VILLACAMPA

Connections between Floer-type invariants and Morse-type invariants of Legendrian knots

MICHAEL B. HENRY

A functional calculus for unbounded generalized scalar operators on Banach spaces

DRAGOLJUB KEČKIĆ and ĐORĐE KRTINIĆ

Geometric formality of homogeneous spaces and of biquotients

D. KoTSCHICK and S. TERZIĆ

Positive solutions for a nonlinear third order multipoint boundary value problem

Yang LiU, Zhang Weiguo, Liu Xiping, Shen Chunfang and Chen HUA

The braid group surjects onto $G_{2}$ tensor space

SCOTT MORRISON

Analogues of the Wiener Tauberian and Schwartz theorems for radial functions on symmetric spaces

E. K. NARAyAnAn and Alladi Sitaram

Semidirect products of representations up to homotopy

Yunhe SHENG and Chenchang ZHU

Homology sequence and excision theorem for Euler class group

YONG YANG 